OPEN ACCESS

Edited by:

Zhi Sheng,

Virginia Tech, United States

Reviewed by:

Marco A. Velasco-Velazquez, National Autonomous University of Mexico, Mexico Robin T. Varghese, Edward via College of Osteopathic Medicine, United States

*Correspondence: Jyotsna Batra jyotsna.batra@qut.edu.au

Specialty section:

This article was submitted to Cancer Molecular Targets and Therapeutics,

a section of the journal Frontiers in Oncology

Received: 06 June 2019 Accepted: 31 October 2019 Published: 26 November 2019

Citation:

Malik A, Srinivasan S and Batra J (2019) A New Era of Prostate Cancer Precision Medicine.

Front. Oncol. 9:1263. doi: $10.3389 /$ fonc. 2019.01263

\section{A New Era of Prostate Cancer Precision Medicine}

\author{
Adil Malik $^{1,2}$, Srilakshmi Srinivasan ${ }^{1,2}$ and Jyotsna Batra ${ }^{1,2 *}$ \\ ${ }^{1}$ School of Biomedical Sciences, Queensland University of Technology, Institute of Health and Biomedical Innovation, \\ Brisbane, QLD, Australia, ${ }^{2}$ Australian Prostate Cancer Research Centre-Queensland, Translational Research Institute, \\ Woolloongabba, QLD, Australia
}

Prostate cancer is the second most common male cancer affecting Western society. Despite substantial advances in the exploration of prostate cancer biomarkers and treatment strategies, men are over diagnosed with inert prostate cancer, while there is also a substantial mortality from the invasive disease. Precision medicine is the management of treatment profiles across different cancers predicting therapies for individual cancer patients. With strategies including individual genomic profiling and targeting specific cancer pathways, precision medicine for prostate cancer has the potential to impose changes in clinical practices. Some of the recent advances in prostate cancer precision medicine comprise targeting gene fusions, genome editing tools, non-coding RNA biomarkers, and the promise of liquid tumor profiling. In this review, we will discuss these recent scientific advances to scale up these approaches and endeavors to overcome clinical barriers for prostate cancer precision medicine.

Keywords: prostate cancer, precision medicine, gene fusion, biomarkers, proteomic technologies, genome editing, non-coding genome, liquid biopsy

\section{INTRODUCTION}

Prostate cancer (PCa) is the second commonly diagnosed cancer (after skin cancer) in the Western society. In 2018, worldwide there were 1.3 million new patients diagnosed with PCa (1). The usage of biomarkers for PCa screening, detection, and prognosis have transformed the diagnosis and management of the disease. Introduction of prostate-specific antigen (PSA) test in clinical practice has ensured early identification and decreased mortality from PCa (2). In spite of its ample value for PCa detection, PSA as a solitary test has some restrictions. The PSA test has specificity and sensitivity ranging from 20 to $40 \%$ and 70 to $90 \%$, respectively, reliant on the applied cutoff for PSA levels (PSA $>4 \mathrm{ng} / \mathrm{ml}$ as normal) (3). One explanation for the poor specificity of the PSA blood test is that some non-cancerous causes may escalate the PSA level in men. For example, benign prostate hyperplasia (BPH) and prostatitis may cause an elevation in PSA levels, and there is little evidence to indicate that $\mathrm{BPH}$ and prostatitis will develop into $\mathrm{PCa}$ (4). Scientists and clinicians are still debating on the use of a single PSA test vs. standard non-screening practices on PCa mortality. A recent study called as cluster randomized trial of PSA testing for PCa (CAP) was conducted in the UK including 419,582 men in 50-69 age range (5). The results indicated no significant difference in PCa mortality of men screened for PSA vs. non-screened patients after a median follow-up of 10 years, but increased early stage low-risk PCa detection. The diagnostic approaches for detecting PCa are changing with advancement in imaging and biomarker discovery to improve early-stage PCa detection. Consequently, despite substantial advances in the exploration of PCa biomarkers, few men are overdiagnosed with inert PCa, while others are missed, developing the invasive disease and diagnosed at a late stage (2). 
Several management options are available for patients ranging from active surveillance for less aggressive PCa to surgery and radiation for advanced disease. Early studies on androgen deprivation in PCa demonstrated the role of androgen receptor in growth and survival (6). Androgen deprivation therapy (ADT) is presently the primary antihormone therapy for treating advanced PCa. Apart from the initial efficacy of ADT, most patients with advanced PCa eventually develop resistance to this therapy and progress to castrate-resistant PCa (CRPC) (7). Antiandrogen drugs such as enzalutamide, abiraterone, and apalutamide have been Food and Drug Administration (FDA)-approved second generation therapies, which has increased survival of CRPC patients (metastatic and localized) $(8,9)$. Alternatively, chemotherapy presents a viable option for treating metastatic $\mathrm{PCa}$ and has appeared to increase survival rate compared to ADT (10). The standard chemotherapy agent, docetaxel, has been used primarily until cabazitaxel was approved in 2010 for CRPC patients (11). In spite of these durable therapies, majority of the cases do not respond to initial therapy due to adaptive resistance, induction of immunosuppressive pathways in the tumor, resulting in tumor relapse (12). An FDA-approved cancer cell vaccine, sipuleucel-T, has been slightly beneficial for increased survival for patients with advanced PCa (13). Several immunotherapy clinical trials for patients with advanced $\mathrm{PCa}$ are still underway and awaiting final outcomes.

Multiple studies have discussed the role of clinically relevant mutations, as well as the level of tumor heterogeneity in primary prostate tumors (14). Genetic tools developed in this "genetic revolutionary" era have been useful to understand this heterogeneity in PCa tumors and identify the finest treatment for patients; new technologies like genomic profiling and use of poly-(adenosine diphosphate) [ADP]-ribose polymerase (PARP) inhibitors like olaparib for patients with mutations in DNA damage response genes have been a boon for precise and effective extrapolation of therapies for individual cancer patients (15). A phase II study by Mateo et al. in CRPC patients, described an increased response of PARP inhibitors to patients with mutations (somatic and germline) in DNA repair genes (16). Improved technologies to interrogate cancer genome have found somatic and germline associations with cancer risk identifying alterations and targets in defined genomic subset of patients. For instance, aberrations in androgen receptor $(A R), T P 53$, retinoblastoma 1 $(R b 1), B R C A 1$, and BRCA2 genes have been evaluated in PCa (17). Somatic mutations in DNA repair genes including BRCA1 and $B R C A 2$ have been reported in PCa patients; wherein BRCA2 mutations $(12 \%)$ were found to be more frequent compared to BRCA1 (2\%) in advanced PCa patients (18). Castro et al. evaluated the status of $B R C A 1 / 2$ in 2,019 patients diagnosed with PCa. They confirmed the presence of BRCA mutations in aggressive phenotype, with poor survival outcomes (19). The same group investigated the influence of $B R C A$ mutations in treatment outcomes in a cohort of 1,302 PCa patients including $67 B R C A$ mutation carriers. The results indicated that $B R C A$ carrier patients undergoing radiotherapy or prostatectomy had shorter survival and developed metastasis sooner compared to non-carriers (20). A recent study identified a germline BRCA2 mutation (c.4211C > G) in a Chinese patient treated with ADT and radiotherapy, the mutation resulting in a truncated protein. The researchers demonstrated that PCa associated with this mutation is sensitive to ADT + radiotherapy and may be effective in patients with this mutation (21).

As the one-size-fits-all approach used in traditional medicine to treat PCa has failed to benefit the patients, the need of the hour is to develop the precision medicine approach which would help patients in the long run. New genomic and proteomic technologies, gene editing technologies, non-coding RNA diagnostics and therapeutics, and liquid tumor profiling have the potential to captivate the promise of precision medicine, highlighting this revolution on different aspects of cancer and their translatability into clinics (Figure 1). In this review, we discuss about the emerging technologies and tools for PCa precision medicine.

\section{GENOMICS AND FUSIONS IN PCa PRECISION MEDICINE}

Genetic influences on PCa have been well-recognized, and our understanding of the molecular genetics of the disease is improving (22). Genetic predisposition could play a decisive role in determining whether a patient should undergo screening and also predict the stage at which the screening may be performed. Early detection of disease and prevention are primary goals for an advancing scientific research community. Genome-wide association studies (GWAS) have been useful in determining genetic risk variants associated with PCa. GWAS involves the investigation of at least hundreds of thousands of variants throughout the genome in large cohorts of individuals, often split into cases and controls, to recognize variants associated with the trait of interest. The most common types of variations in the human genome are termed single nucleotide polymorphisms (SNPs) and are believed to directly contribute to the progression of many complex diseases, including PCa (23).

Numerous advances in high-throughput genotyping have improved the performance of GWAS and even more recently detailed whole-exome and whole-genome sequencing studies. Currently, more than 150 loci were reported to be associated with PCa susceptibility and aggressiveness that accounts for $\sim 40 \%$ of PCa risk $(21,24)$. Risk information could be collected and analyzed using an array of SNPs and estimate an individual's risk of developing a disease (25). This risk is calculated by sum of all the risk SNPs corresponding to a phenotype, to the effects of GWAS on the same phenotype. This prediction of polygenic risk could improve clinical decisions in screening for PCa (26). Using this model of polygenic risk score (PRS)/polygenic hazard score (PHS), men at high cancer risk could be identified thus, reducing morbidity and mortality. Recent studies have suggested that polygenic risk profiling on the basis of PRS could provide personal and clinical utility to patients and health management $(27,28)$. These risk variants have been used to determine a PRS estimating an individual's risk to developing disease $(29,30)$. In a recently published research study, Seibert et al. calculated PHS for age-related risk from SNPs, which predicted diagnosis of aggressive cancer (31). The study used data from 31,474 men 


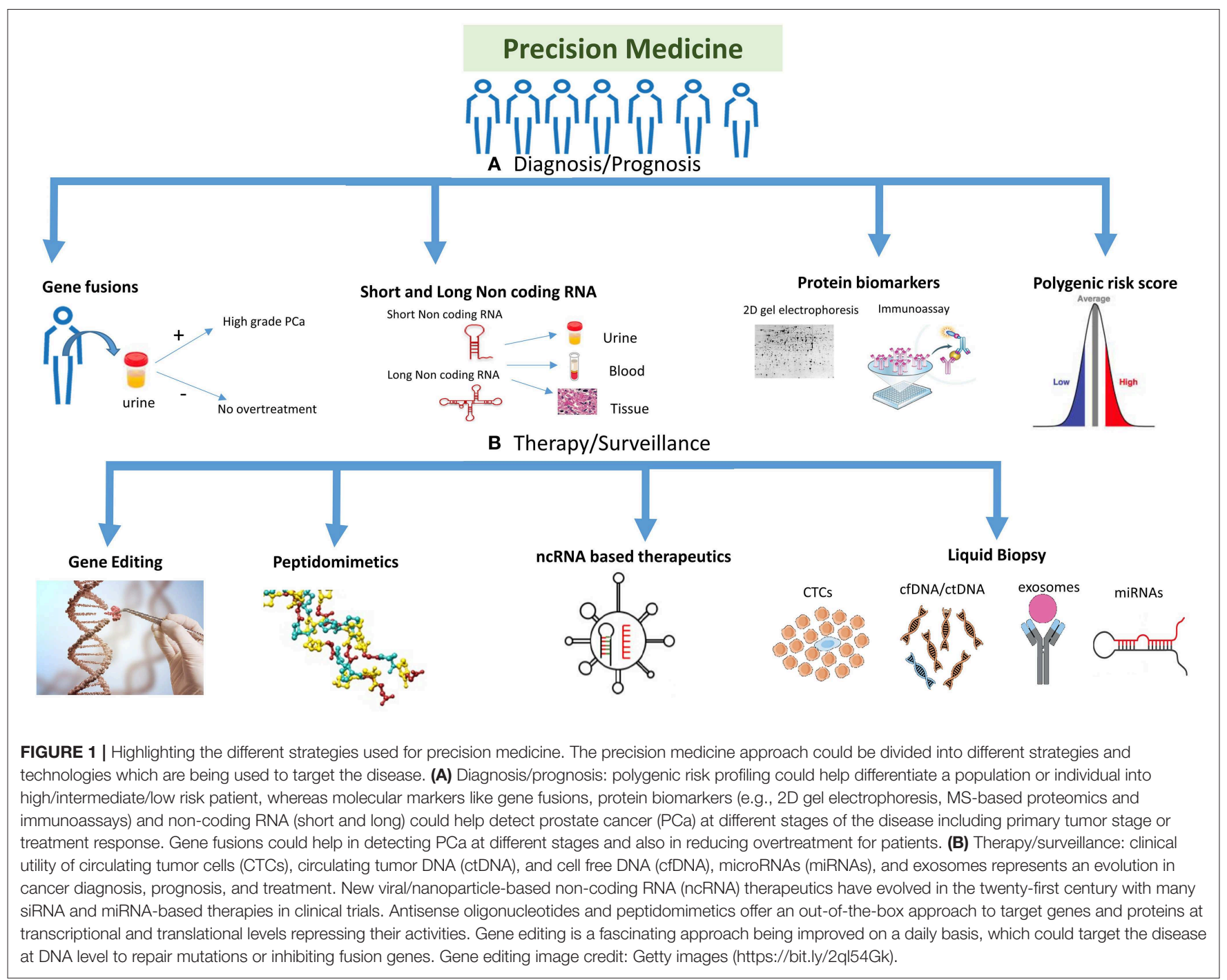

of European ancestry, which had a total of 201,043 SNPs for analysis. In a validation set of 82,429 men, 54 SNPs predicted a higher score for aggressive cancer. Moreover, increased PHS led to a higher prediction for aggressive PCa with PSA screening.

PSA screening test has led to high false positives with overtreatment of benign disease. PRS has been observed to aid in identifying men with elevated PCa risk attaining greater benefit/risk ratio compared to PSA testing $(31,32)$. Men in the top $50 \%$ with PRS scores account for $76 \%$ of PCa with the top $20 \%$ individuals accounting for $42 \%$ of aggressive PCa (31). PRS does not directly correspond to aggressive PCa but solves the false-positive PSA screening and associated overtreatment of disease (33). Additional clinical risk factors with well-developed active surveillance may help decide if treatment is needed for PCa (34). This PRS-regulated PCa screening would lead to individuals being recommended screening at specific age. A recent investigation by Schumacher and colleagues identified 63 novel variants associated with PCa susceptibility, with the authors claiming that the findings could improve risk prediction using PRS risk assessment with informed screening and help disease management (35). A team led by Lecarpentier et al. suggested that PRS score can be useful determinant for the management of breast or PCa risk in BRCA1/2 mutation carrier men. At 80 years of age at the 5th and 95th percentiles of the PRS varied from 7 to $26 \%$ for carriers of BRCA1 mutations and from 19 to $61 \%$ for carriers of BRCA2 mutations, respectively. They evaluated prostate and breast cancer risk using PRS based on 88 female breast cancer and 103 PCa susceptibility variants (36). Although this study gives an improved screening strategy for mutation carriers in whom PRS prediction can be used for reducing over diagnosis in general population, it lacks in assessing the effect of family history with mutation carriers associated with PRS. The core philosophy of precision medicine is personalized management of disease. Recent studies in coronary heart disease have invoked the potential of PRS in therapeutic interventions, with the use of proper dosage of statins in individuals who have at least one risk factor for cardiovascular problem (26). Genetic risk scoring has shown promise in 
identifying individuals who may profit from early preventive actions and disease management. However, uncertainty looms large on the risk estimates of individuals screened using PRS vs. familial disease trait, with its inability to calculate variants not related to causal genetic factors leading to apprehensions in estimation of risk (37). The discrepancy in using this risk score in different populations is also a major concern to transfer it to clinical settings (38). This risk score bias needs to be validated in a diverse population to remove the effects of demography. These precincts should be looked upon by researchers with developing new methods to improve generalization of PRS. New statistical models to identify and characterize allele frequency in unidentified locus associated with disease would help in calculating genetic risk of an individual with more precision.

Gene fusion is a phenomenon wherein a hybrid gene is formed from two genes by chromosomal inversion, translocation, or deletions (39). Emerging experimental data indicate that gene fusions are important molecular events in the growth and advancement of PCa (40). The most common gene fusions in PCa genomes are with members of the ETS family of transcription factors, such as ERG and ETV genes (41). Assessing ETS status, and developing assays, therefore could probably be a way forward. Immunohistochemistry (IHC) has been a standardized tool to evaluate ETS status in clinical settings; however, this technique falls short of detecting fusions involving other ETS family members. To overcome this problem of characterizing prostate tumor ETS status in clinical samples, Tomlins et al. developed a method, clinical laboratory improvement amendments decipher assay (42). The assay achieved $91 \%$ sensitivity and $98 \%$ specificity in detecting ETS status when validated in 252 primary prostate tumors and independent 155 radical prostatectomy samples. Torres and colleagues further evaluated this assay to assess ETS status in the Decipher Genomics Resource Information Database transcriptome database, expanding the validation by including ETV1, ETV4, ETV5, and ERG (43). The model showed 100\% robustness among samples for ETS status, with ETV1, ETV4, and ETV5 showing area under the curve (AUC) of 98, 88, and 99 , respectively. This approach emerges as an individualized tool for molecular classification of PCa tumors utilizing ETS gene fusion status. This model, when applied to radical prostatectomy samples, predicted m-ERG model sensitivity at $93 \%$. However, in uncommon cases of ETV5 overexpression, this model had a lower sensitivity. In spite of the advantages of this assay, the presence of ETS rearrangements was not associated with adverse outcomes in one of the cohorts used by the group contrasting its previously published data (44). Henceforth, the significance of this data seems unclear at the moment, but the authors argued about the use of this classifier assay as a tool for molecular classifying prostate tumors.

The most common fusions studied in PCa are in ERG, which belongs to ETS family. The early twenty-first century saw the discovery of genomic rearrangements in TMPRSS2 and $E R G$ oncogenes in PCa. Later in the decade, it was identified and accepted that $\sim 50 \%$ of the tumors in prostate harbored TMPRSS2-ERG fusions as the most recurrent genomic modification (45). These rearrangements have been observed in early $\mathrm{PCa}$ representing high Gleason Score with poor prognosis as a result of ERG-modulated transcription events affecting PCa cells invasiveness, migration, and epithelial-tomesenchymal transition (EMT) (46). These fusion genes were detected previously using fluorescence in situ hybridization (FISH) or the traditional PCR techniques. Several new in silico tools including SOAPfuse, FusionMap, JAFFA, FusionSeq, and GFusion have been developed to identify novel fusion genes using RNA-seq data. These computational tools have presented an alternative platform compared to traditional methods like reverse transcription PCR and FISH to detect fusions with higher sensitivity and a lower false-positive rate (47). It has been suggested that $E R G$-targeted drug development in combination with urine tests available to detect TMPRSS2-ERG fusion could define a precision medicine procedure for $\mathrm{PCa}$ patients $(48,49)$. A clinical trial conducted by Jewish General Hospital, Quebec, Canada, recruited 65 high-risk PCa patients treated with radiation and hormonal therapy after biochemical failure to evaluate the predictive value of TMPRSS2-ERG gene fusion (ClinicalTrials.gov Identifier: NCT02588404). This study once completed will correlate Gleason Score and T-stage with TMPRSS2-ERG gene fusion event. In addition, researchers have also looked at targeting ERG directly; ERG knockdown in VCaP CRPC cell line indicated the role of ERG in proliferation and blocking differentiation of prostate cells to neuroendocrine and luminal cell types (50). These findings by Mounir et al. supported the clinical utility to target these alterations.

A potential therapeutic strategy involving inhibition of ETS cofactors including DNA-dependent protein kinase, PARP1, and histone deacetylase 1 rather than the fusion has generated a lot more interest in the scientific community (51). A randomized phase I and II studies inhibiting PARP1 in CRPC patients were started in 2012 (ClinicalTrials.gov Identifier: NCT01576172). Primary results showed that the addition of veliparib (PARP inhibitor) to abiraterone acetate plus prednisone therapy did not show any response (52). Similarly, phase II trials targeting HDAC in CRPC patients have been completed with disappointing results $(53,54)$. These results do show that targeting transcription factors does need a novel strategy. A lack of "new-age" compounds targeting ETS factors makes it a tedious task to target these oncoproteins for developing new therapeutics for PCa. There have been advancements in developing small molecule antagonists to ERG; YK-4-279 was discovered as a small molecule targeting FLI1 protein, a homolog of ERG. Its derivative, TK216 is currently in phase I trial and is anticipated to have considerable effect on developing ETS-targeted therapies (55). Computer-aided drug discovery approach has yielded a small molecule compound VPC-18005 that directly targets ERG at low concentrations suppressing metastasis of ERG-expressing PCa cells (56). Wang et al. devised a new approach for targeting oncogenicity of ERG using inhibitory peptidomimetics. The group screened a phage display peptide library, identifying peptides that specifically bind to ERG protein but not to a negative control protein. These ERG-inhibitory peptides (EIP) disrupted ERG-ETS domain/DNA interactions via binding to ETS domain of ERG (57). The observations in this research wherein EIP destabilizes ERG may provide an alternative 
approach targeting transcription factor for therapeutic purposes. Peptidomimetics offers target specificity with low cytotoxicity and side effects, but vital questions like high developmental costs, delivery, and permeability of the membrane needs to be answered before clinically developing peptides for PCa treatment. While this complex strategy offers specificity and efficient target affinity, issues still remain about solubility, stability, delivery systems, and high cost of development. Future studies should concentrate on overcoming these limitations and developing new inhibitors of ERG to treat resistant and metastatic form of PCa.

In addition to TMPRSS2-ERG/ETS fusion in PCa, new fusion transcripts have been identified in both normal and tumor prostate tissue (58). Zhao et al. identified 21 fusion transcripts that are involved in $\mathrm{PCa}$, out of which 13 were novel fusion transcripts (59). These included fusion transcripts between protein coding genes in addition to long intergenic noncoding RNA (lincRNA) as fusion partners in some transcripts. The fusion transcripts ACSS1-APMAP, RP11_17A19.1-KCTD1, and ZNF841-ZNF432 were found to be highly expressed in tumors vs. benign tissues. The authors found lincRNA fusion to be the most common in PCa, highlighting the biological importance of lincRNA fusion transcripts in tumorigenesis (59). Similarly, Qin et al. found SLC45A3-ELK4 fusion RNA in PCa cell lines, functioning as a chimeric lincRNA regulating cell proliferation (60). At this point of time, this is a new mechanism to understand the biology of lincRNA fusions in PCa. However, if this mechanism can be elucidated, silencing of these genes using clustered regularly interspaced short palindromic repeat (CRISPR)-CRISPR-associated (Cas) can be achieved. It still remains to be seen how effective are these lincRNA fusions in detecting PCa or targeting them as a therapy.

Lai et al. investigated the mechanisms of fusion gene transcription/splicing from RNA-seq data of prostate tumors and cells using a program FusionMap (61). It was an interesting computational analysis wherein the authors indicated a concurrence of GT-AG intron donor-acceptor splice site in $76 \%$ of fusion junctions. In addition, the prediction indicated that non-fusion splice sites and fusion junctions have similarity in hybridization. Some new fusion transcripts were detected in androgen-/antiandrogen-treated cells highlighting the importance of fusion genes in PCa. C1QTNF3-AMACR fusion transcript was found to have an expression profile distinct from their parental genes in prostate tumors. Out of 185 newly identified fusion transcripts, some fusion genes including SIDT2-TAGLN, HARS-ZMAT2, CTBS-GNG5, NOS1AP-c10rf226, and DHRS1-RABGGTA were differentially expressed in prostate tumors as detected in clinical RNA-seq dataset. This uniqueness of cancer driver gene fusions in tumor cells highlight their importance for therapeutic application targeting cancer cells.

Phosphatase and tensin homolog (PTEN) is a tumor suppressor gene associated as a genomic marker in various oncological settings. Aberrations in PTEN are found to be most common in PCa wherein inactivation and deletion have been identified in prostate tumor patients at radical prostatectomy stage and in CRPC (62). PTEN loss has been positively correlated with the TMPRSS2:ERG fusion event, implying roles of both these somatic events in prostate tumorigenesis (63).
Clinical studies indicate the association of patient's PTEN status with effective therapies. Robust clinical assays like IHC and FISH have enabled clinicians to use PTEN as a prognostic marker in tissue and liquid biopsies (64). PTEN loss has been correlated with increased activation of phosphoinositide 3-kinase-RAC-alpha serine/threonine-protein kinase pathway, which is concomitant with adverse clinical outcomes. These pathways are being tested clinically with new therapeutic compounds in metastatic PCa patients (64). Inactivation or loss of PTEN genes leads to PI3K pathway activation leading to tumorigenesis. Recently, Mateo et al. tested a drug, GSK2636771, for the selective pathway inhibition of PI3K $\beta$ (65). The group evaluated different parameters including antitumor activity and the pharmacokinetic and pharmacodynamic properties of this drug and concluded clinical benefit of GSK2636771 as a selective inhibitor of PI3K pathway in patients with solid tumors.

All the studies mentioned in this section are still trying to develop and target these fusion genes; some are currently in development. The status of these fusion genes can be a guidance during therapy surveillance, risk stratification, or as effective target therapies. The detection of this genes and their fusion partners may be effective in prediction of hormonal treatments and classify them as androgen dependent or independent, which could alter therapy stages and improve outcomes. The new approach of peptidomimetics to target these genetic anomalies could be the answer to effectively reduce tumor growth without changing the normal cellular functions. However, before this can be used as precision therapies, modifications to change the peptides needs to be done for proper intake into the cells and inhibit these fusions.

\section{PROTEIN BIOMARKERS AND NEW PROTEOMIC TECHNOLOGIES}

Proteomic technologies and proteomics have always been a fascinating area of research for the discovery of cancer-specific biomarkers, improvement in prognosis, and treatment response biomarker identification, contributing to in-depth understanding of cancer pathology and developing new effective therapies (66). Mass spectrometry (MS) has been the forefront for proteomic and peptidomic analysis, identifying thousands of proteins and translating relevant data to clinics. In the past decade, different MS-based platforms like liquid chromatographytandem MS (LC-MS), two-dimensional fluorescence difference gel electrophoresis (2D-DIGE), matrix-assisted laser desorption ionization (MALDI)-time of flight MS, capillary electrophoresisMS, selected reaction monitoring (SRM)/multiple reaction monitoring (MRM), and parallel reaction monitoring (PRM) have been developed for biomarker discovery, paving the way for translating it to clinically relevant biomarkers in prognosis and diagnosis.

A number of relevant biomarkers (Table 1) and multimarker assessment tests are now available for clinicians to make the difficult clinical decision of treating PCa. Prostate Health Index is a blood test for PCa detection, which involves combining total PSA, free PSA, and proPSA. This test was seen to be discrete 
TABLE 1 | Different studies highlighting the role of proteomic techniques in precision medicine.

\begin{tabular}{|c|c|c|c|}
\hline Method & Study & Sample & References \\
\hline Quantitative proteomics & $\begin{array}{l}\text { Prediction of disease aggressiveness via } \\
\text { proteomic biomarkers }\end{array}$ & Formalin-fixed paraffin-embedded tissue & $(71)$ \\
\hline MALDI-MS profiling & $\begin{array}{l}\text { Potential of } \beta \text { microseminoprotein } \\
\text { combined with PSA as biomarkers }\end{array}$ & Post-DRE urine samples & $(74)$ \\
\hline $\begin{array}{l}\text { 2D gel electrophoresis } \\
+ \text { MALDI-MS }\end{array}$ & $\begin{array}{l}\text { Urinary protein changes after radical } \\
\text { prostatectomy }\end{array}$ & Urine samples postradical prostatectomy & $(78)$ \\
\hline 2D-DIGE/MS & $\begin{array}{l}\text { Panel of diagnostic biomarkers } \\
\text { ( } \alpha \text {-1-microglobulin, transferrin, and } \\
\text { haptoglobin) }\end{array}$ & Urine samples & (79) \\
\hline iTRAQ & Multiplex biomarker panel for diagnosis & Serum and urine & $(80)$ \\
\hline iTRAQ + SRM/MRM & $\begin{array}{l}\text { Proteomic analysis of urinary extracellular } \\
\text { vesicles from high grade PCa }\end{array}$ & Urinary extracellular vesicles & $(81)$ \\
\hline $\begin{array}{l}\text { ELISA + Western } \\
\text { blotting }\end{array}$ & $\begin{array}{l}\text { Immunoassay-based validation of urinary } \\
\text { exosomal proteins as PCa biomarkers }\end{array}$ & Exosomes in urine & (83) \\
\hline $\mathrm{IHC}+\mathrm{SRM}+\mathrm{PRM}$ & $\begin{array}{l}\text { Verification of urinary biomarkers using } \\
\text { targeted proteomics }\end{array}$ & Urine & (84) \\
\hline
\end{tabular}

and surpass its singular components with additional aspects of predicting progression of PCa during active surveillance (67). Alternatively, PROSTARIX ${ }^{\mathrm{TM}}$ is a commercially available urinary test by Metabolon Inc. This test measures a panel of four metabolites (sarcosine, glutamate, alanine, and glycine) by chromatography and MS after digital rectal examination (DRE) (68). This test showed increased specificity and sensitivity vs. serum PSA (AUC 0.78). Another test named ConfirmMDx, an epigenetic test for predicting prostate biopsy using prostate core samples, helps patients in managing PCa at initial diagnosis. A patient is informed about the need for rebiopsy using this test with a sensitivity of $62-68 \%$ and a specificity of $64 \%$ (69). The Matloc study confirmed the usage of this test to reduce the need for biopsy in many patients with a negative predictive value of $88-90 \%(70)$.

Other tests have been developed for identifying aggressive disease that can reduce prostate biopsies. An eight-biomarker signature [pS6 (phosphorylated S6), HSPA9, DERL1, PDSS2, YBOX1, CUL2, FUS, SMAD4] tissue-based proteomic assay was developed by Metamark genetics for formalin-fixed tissue biopsies for precision in clinical decision after biopsy. This assay using proteomics looks promising, but efficacy and reproducibility using formalin-fixed paraffin-embedded tissues vs. fresh tissues need further validation $(71,72)$. Many research groups worldwide are adopting different protein profiling approaches for developing new assays for PCa diagnosis and prognosis $(73,74)$. Flatley et al. collected pre- and post-DRE urine samples from PCa and other prostatic disease patients and performed MALDI-MS profiling. This approach found evidence of $\beta$-microseminoprotein (MSMB) to be a potential biomarker in PCa diagnosis. When combined with serum PSA levels, the sensitivity of MSMB/PSA model gave increased sensitivity of $96 \%$ at $26 \%$ specificity (74). This model is still insufficient for population screening due to low specificity; however, including MSMB in a large panel of biomarkers would justify its inclusion and reduce the number of needless biopsies in benign patients.

A research was conducted in 2015 called The Stockholm (STHLM3) study to improve the performance of detecting
PCa, wherein a combination of genetic tumor markers (232 SNPs), PSA concentration, plasma proteomic markers (MIC1, MSMB, intact-PSA, free-PSA, and HK2), and standard clinical variables (prostate volume, family history, age, DRE, and previous biopsies) were tested in a cohort of Swedish men aged 50-69 years. The primary aim of the STHLM3 study was to assess the efficacy of this model to increase specificity for detecting PCa. An improved AUC (0.74) compared to standard PSA (0.56) was observed identifying tumors with the STHLM3 model. All the variables used in the study were found to be significantly associated with Gleason Score of at least 7 in multiple logistic regression model. This approach also showed a decrease in benign biopsies by $44 \%$ (75). This model clearly outperforms the PSA as a screening tool for PCa. The STHLM3 model was updated wherein intact PSA has been removed and a new biomarker, the rare germline mutation (G84E) in $H O X B 13$, has been added to improve this test (76) (ClinicalTrials.gov identifier: NCT03639649). It was found that using this updated test, biopsies were reduced by $34 \%$ compared to using PSA alone. This test has also been validated in an independent clinical setting in Norway and Sweden, which showed its efficacy in reducing biopsies in a substantial amount (77). In addition, studies validating this test in non-Caucasian population will commence shortly. Although this test has been found to be clinically better than PSA, there is yet no evidence of it being beneficial to men above 70 and below 50 years old. At this juncture, the STHLM3 test still seems to be the best possible option for patients in the 50- to 69-year-old category.

Owing to the complexity of PCa tumor and its heterogeneity, a signature or a panel of biomarkers could be a more practical and plausible route to the diagnosis and prognosis of this cancer. Three unique proteins, namely, cyclin-dependent kinase 6, Galectin-3-binding protein, and L-lactate dehydrogenase $\mathrm{C}$ chain, were identified in urine samples using two-dimensional gel electrophoresis coupled with MALDI-time of flight MS related to surgical margin status after radical prostatectomy. These proteins may assist in evaluating tumor progression after 
surgical treatment (78). This research on PCa urinary proteome identified differences in positive and negative surgical margin patients after radical prostatectomy, highlighting the importance of combining genomic and proteomic approaches to understand PCa status in patients. Davalieva et al. accounted for the potential of three biomarkers ( $\alpha-1$-microglobulin/bikunin, transferrin, and haptoglobin) in urine using 2D-DIGE/MS. All three proteins yielded different specificity and sensitivity, but the integration of haptoglobin and $\alpha$-1-microglobulin/bikunin had higher accuracy in PCa detection compared to PSA underlying its potential as a urine based biomarker (79). Quantitative proteomic (MS-based) approaches like isotope-coded affinity tag and isobaric tag for relative and absolute quantitation (iTRAQ), which are isobaric labeling methods, have been used recently without the need of traditional gel electrophoresis methods. Using iTRAQ, Zhang et al. identified three proteins, namely, serum platelet factor 4 variant 1 , urinary cysteine-rich secretory protein 3 , and PSA for precise diagnosis of PCa. The AUC of this three panel of proteins was higher (AUC, 0.941) compared with PSA alone (AUC, 0.757) for PCa prediction. Platelet factor 4 variant 1 and cysteine-rich secretory protein 3 when combined could attain better discrimination in gray zone of PSA $(4-10 \mathrm{ng} / \mathrm{ml})$ and had the potential to differentiate between high-grade prostatic intraepithelial neoplasia and PCa (80). Fujita et al. found five proteins when analyzing urinary extracellular vesicles using iTRAQ and LC-MS/MS to be better predictors of PCa compared to PSA from high Gleason score PCa (81). iTRAQ coupled with 2D-LC-MS/MS was used by Katafigioti et al. and team for the proteomic profiling of PCa tissue. They detected potential protein biomarkers in PCa tissue, namely, secreted protein acidic and rich in cysteine, glutathione peroxidase 3 precursor, zinc alpha 2 -glycoprotein, cofilin-1, and heat shock protein-90 $\beta$. When investigated in urine samples, zinc alpha 2 -glycoprotein was found to be discriminative in early diagnosis of PCa (82).

A group of researchers led by Wang et al. described the prospectus of urine exosomal proteins as biomarkers and problems faced when validating these MS-based results using clinically relevant techniques such as enzyme-linked immunosorbent assay (ELISA) and Western blotting. The group observed that ELISA analysis of combined flotillin 2 and Parkinsonism associated deglycase (PARK7) had a value of 68\% sensitivity and $93 \%$ specificity, distinguishing PCa patients with healthy controls. Western blotting analysis for flotillin 2, late endosomal/lysosomal adaptor, MAPK and MTOR activator 1 (LAMTOR1), transmembrane protein 256 (TMEM256), and RAS-associated protein 3B (RAB3B) proteins showed higher expression of these proteins in urinary exosomes of PCa patients compared to healthy patients (83). Validations in a higher number of patient samples may improve their utility as potential biomarkers in clinical settings.

Proteomic-based discovery of biomarker does not always translate to a diagnostic utility. Validation of biomarkers in a large pool of patient samples is an important step after the discovery phase studies have been conducted. The primary challenge in targeted proteomics approach for biomarker discovery and validation is arduous assay optimization. A prevalidation workflow was developed by Adeola et al. to clinically validate biomarker discovery. This bioinformatics verification platform used IHC and SRM verification as a preliminary step. The proteotypic peptides successful in this step were validated by PRM. The results revealed 12 potential biomarkers differentiating PCa with healthy control samples (84). This pipeline serves as better validation tools compared to Western blotting and IHC analyses.

Similarly, shotgun proteomics via data-dependent acquisition (DDA) has assisted in MS detection, but the problem of missing data with data acquisition has haunted the area of discovery proteomics (Figure 2). Several data processing tools and spectral libraries are being developed to overcome this problem (86). Sequential window acquisition of all theoretical fragment-ion spectra-MS is now being used in many research labs for biomarker discovery wherein the combination of DDA and data-independent acquisition (DIA) is being applied to construct spectral libraries, giving a broad coverage of all precursor ions within a particular $\mathrm{m} / \mathrm{z}$ range (87). This precise and reproducible quantification pipeline shows promise in $\mathrm{PCa}$ biomarker discovery in the future. In a recent study using integrative proteomics in $\mathrm{PCa}$, sequential window acquisition of all theoretical fragment-ion spectra-MS was used to identify novel molecular pathways with the proteome of $\mathrm{BPH}, \mathrm{PCa}$, and CRPC patients. The proteomic data identified changes in tricarboxylic acid cycle, which was comparatively altered in CRPC vs. $\mathrm{PCa}$ and $\mathrm{PCa}$ vs. BPH patient samples. More than 3,000 proteins were quantified in these clinical sample batches with importance of proteins in mechanisms supporting $\mathrm{PCa}$ growth and progression. These study provided an integrative robust analysis that underlying proteomic changes are not always related to copy number, RNA expression, and DNA methylation (88). With respect to precision medicine for $\mathrm{PCa}$, these highthroughput proteomic technologies will hold greater value in disease management and improved prognosis and diagnosis. In addition, for personalized medicine for $\mathrm{PCa}$, proteomic signatures will be more helpful in achieving the ambitious goal of diagnosis and treatment outcome prediction. Technological advances in this field have led to development of new methods to screen and generate multiple candidate protein biomarkers with the help of LC-MS/MS. Multiple research has identified various candidate biomarkers, but a greater concern still holds on the verification and validation of these selective biomarkers. MRM approach can be used to develop assays providing absolute quantification of protein biomarkers in clinical samples. Using this method, Fortin et al. showed clinical quantitation of PSA with concentrations from 4 to $40 \mathrm{ng} / \mathrm{ml}$ showing good correlation with ELISA tests (89). Furthermore, this technique should be used in new protein biomarkers identified in large cohort of patients to validate the efficacy of the biomarkers to be used in clinical settings. In addition, the use of protein biomarker panels needs to be explored more using MS-based techniques, which could lead to increased sensitivity and specificity. This kind of pattern studies have now been used in several publications, but the pretext of proteomics in discovery and verification of these panels needs to be fulfilled in personalized medicine for the prediction of patients' response to therapy, diagnostic, and prognostic purposes. 


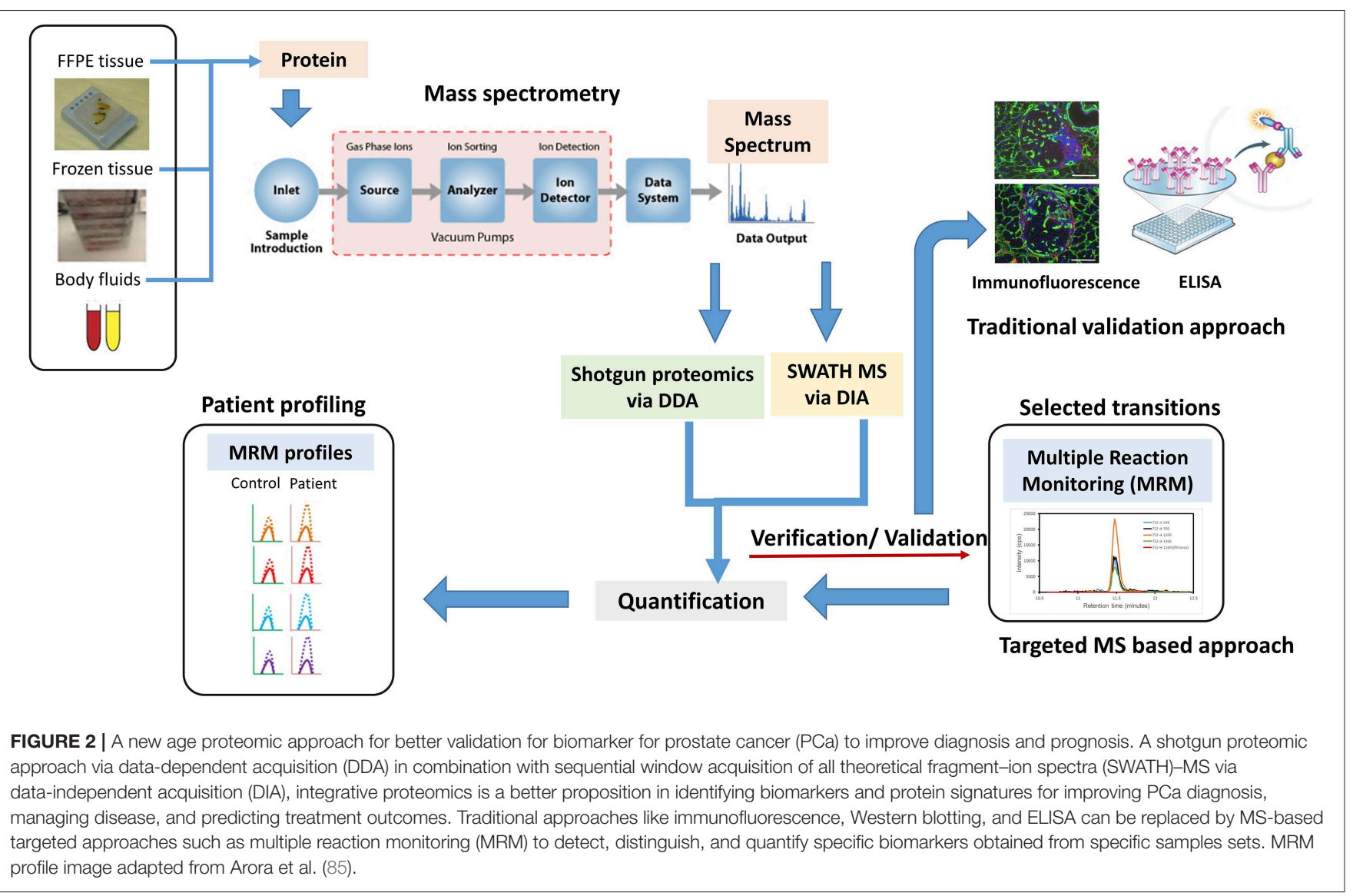

\section{GENOME EDITING FOR PRECISION THERAPY}

Efforts to treat genetic diseases have always been an area of development and contextualization. Gene editing, redefined and rediscovered, provides an innovative approach to increase gene correction and develop a precision therapy transforming clinical technologies. There are some genetic tools existent for this objective, comprising of zinc-finger nuclease, transcription activation-like element nuclease, and the latest, CRISPR/CRISPR-associated (Cas) system. The latter is more cutting edge due to ease in generation and immense efficacy for gene targeting, engaged to effectively accomplish knockouts in different human cell lines (90). Proficiency, precision, and highthroughput screening demonstrate that CRISPR/Cas9 editing technique can be contemplated for therapeutic applications. The first CRISPR phase I clinical trial started in 2016 in China, wherein CRISPR/Cas9 is performed ex vivo to knockout programmed cell death protein-1 in T cells (NCT02793856). These engineered cells have been selected and infused into patients. Similar clinical trials targeting programmed cell death protein-1 on PCa (NCT02867345), renal cell cancer (NCT02867332), bladder (NCT02863913), and esophageal cancer (NCT03081715) have also been started.

Genome-editing technology targeting chromosomal breakpoints could provide an alternative methodology treating human cancers via inhibiting fusion genes. Herpes simplex virus type 1 thymidine kinase (HSV1-tk) is a prodrug converting enzyme that forms thymidine monophosphate by phosphorylating thymidine, an active ingredient in DNA synthesis mechanism. HSV1-tk, unlike its mammalian equivalent, also phosphorylates ganciclovir (prodrug), a synthetic nucleoside homolog (91). The phosphorylation step leads to elevated ganciclovir monophosphate levels in mammalian cells. This monophosphate is modified to triphosphate, which blocks DNA synthesis. Cells are immune to this phenomenon lacking HSV1-tk due to failure in phosphorylating ganciclovir. Chen et al. using a mutated Cas9 used CRISPR to insert HSV1-tk in the chromosomal breakpoints of TMEM135-CCDC67 and MAN2A1-FER fusion genes. This led to cell mortality in cell culture system and reduction in tumor mass and mortality in mice grafted with human prostate and liver cancers (92). The research did not observe any major cytotoxicity in cell or animal models. The authors discussed a castrate-resistant improved methodology of genome editing with tumor immunotherapy and other therapeutic treatments when obligatory for improved therapeutics. Although this study reduced the cytotoxicity, a major issue concerning CRISPR models has been non-specificity. The "unknown" stress induced by CRISPR on the genome via development of new polymorphisms could lead to consequences not intended and ultimately producing a negative effect. The size of Cas9 protein has always been an issue that has resulted in 
unintended editing in the genome. New Cas proteins have been identified, which are smaller in size. In a recently published study in Nature, a group of researchers from University of California have revealed a distinct genome editing platform and termed as CRISPR-CasX system (93). The authors, through cryo-electron microscopy, elucidated the structure of CasX in its assembly state determining its small size and DNA cleavage properties. This protein is derived from non-pathogenic microbes found in groundwater and sediment. The additional property of nonimmunogenicity makes it an ideal genome editing tool compared to Cas9 and Cas12a. Once this system gets developed in humans, it would provide opportunities for therapeutic delivery, and the notion of safety can be put to rest.

GPRC6A, a G-protein-coupled receptor, has been found to be a sensing receptor promoting the progression of $\mathrm{PCa}$ and a target for developing antagonists to treat $\mathrm{PCa}$. Ye et al. used CRISPR-Cas9 technology to delete GPRC6A in human PC3 cells and observed a diminishing reaction to L-Arg, osteocalcin, and testosterone stimulation of ERK, Akt, and mTOR phosphorylation with reduced cell proliferation and downregulation of OCN, PSA, MMP9, BMP3, RUNX2, and $V E G F$ genes, which are involved in PCa progression (94). This study indicated that GPRC6A editing could reduce expression of enzymes regulating intratumor androgen biosynthesis. Additional efforts to develop antagonist against this target could lead to development of new treatments for PCa. A focal limiting feature in the development of CRISPR in clinical trials is the selection of the delivery method. Developing a delivery system for therapeutic targeting via CRISPR/Cas9 requires specific administration and formulation of the delivery reagent. Zhen et al. established an aptamer-liposome centered CRISPR/Cas9 chimera, which recognizes prostate-specific membrane antigen (PSMA) receptor expressed on PCa cells. The team designed chimeras with RNA Aptamer A10, which have the capability to bind to PSMA. The research suggested A10-liposome-CRISPR/Cas9 chimera delivery system as an appropriate method targeting tumors in vivo, exonerating its usage as a viable therapeutic treatment method for human PCa (95). Evaluation of delivery methods and potential genotoxic effects of using CRISPR can only be done in an efficient manner when additional preclinical studies are implemented. There is no guarantee of therapeutic efficacy even after developing new delivery methods of CRISPR vectors. In the coming time, whether CRISPR makes a difference in PCa or not is up for debate but is definitely worth the effort.

\section{NON-CODING-RNA-BASED DIAGNOSTICS AND THERAPEUTICS}

Non-coding RNAs (ncRNAs) are generally distributed into dual primary groups in view of their sizes: small ncRNAs $(<200$ bp) and long ncRNAs (lncRNA, >200 bp) (96). Small ncRNAs primarily comprise of circular RNAs (circRNAs), microRNA (miRNA), small nuclear RNA (snRNA), and small interfering RNA (siRNA), besides transfer RNA (tRNA), and ribosomal RNA (rRNA) (97). LncRNAs are divided into inter- and intragenic
lncRNAs vis-à-vis their positions in the genome comparative to protein coding genes (98); intragenic IncRNAs can be additionally categorized as exonic, intronic, and overlapping lncRNAs (98). Non-coding RNAs such as IncRNAs, miRNAs, and circRNAs have demonstrated lineage-specific patterns, which could be used as biomarkers to discriminate between different tissue types (99). This section will discuss the different preclinical and clinical aspects of ncRNAs like miRNAs, circRNAs, and lncRNAs, which have been well researcherd in PCa.

MicroRNA plays an important role in intracellular processes regulating gene expression at the level of transcription binding to regulatory elements. Several miRNAs have been screened for potential biomarkers (Figure 3) for disease aggressiveness and therapeutic resistance, as well as therapeutic targets (100). miRNA profiles have been studied to identify differences between $\mathrm{BPH}$, localized, and metastatic form of PCa. In a study by Lichner et al., miRNA downregulation was observed in higher Gleason grade prostate tumors compared to lower Gleason grade tumors (101). The same group pointed out that lower levels of miR-331-3p and miR-152 in patients were associated with increased risk of biochemical failure (102). A recent study analyzed circulating microRNAs in patient serum and correlated them with clinicopathological characteristics (103). The authors identified miR-375, miR-106b, miR-21, and miR141-3p to have increased expression in PCa patients compared to healthy controls. This study discussed the use of miR-375, miR-141-3p, and miR-21 as potential biomarkers, with significant improvement in prediction of $\mathrm{PCa}$ in patients. In a review by Matin et al., the diagnostic and prognostic potential of miRNAs in $\mathrm{PCa}$ were briefly reviewed wherein different aspects of miRNA expression pattern in tumor tissue and circulating miRNA were discussed in length. The authors also deliberated on the polymorphic aspect of miRNA and how these polymorphisms can predict an individual's response to miRNA therapy (104). Although these findings may shed light on the potential of miRNA as diagnostic, prognostic markers and its therapeutic potential in $\mathrm{PCa}$, reproducibility and validations in large cohorts limit their usage as biomarkers for PCa. Another area of deliberation is the detection of circulating miRNAs in cancer. miRNA detection has been possible by quantitative reverse transcription PCR, next-generation sequencing, microarray, and biosensor-based assays (105-107). Although these strategies have improved detection of miRNAs, an area of contention remains in low abundance of these molecules in serum or plasma and the cost of using these technologies in a clinical setting. Despite various obstacles, targeting miRNA function has been achieved, which gives a clear indication of targeting these molecules for PCa precision medicine.

The purpose of expediting miRNAs as targets for anticancer therapeutics is their dysregulation in different cancers targeting several genes and their capacity to modify phenotype (108). Various strategies ranging from using CRISPR, miRNA sponges, antisense oligonucleotides to small molecule inhibitors have been used to target miRNA expression (104). High-throughput sequencing has revealed miRNA changes in PCa patients, correlating its diagnostic value as a therapeutic target (109). Antisense oligonucleotide (ASO) based therapeutics have been 


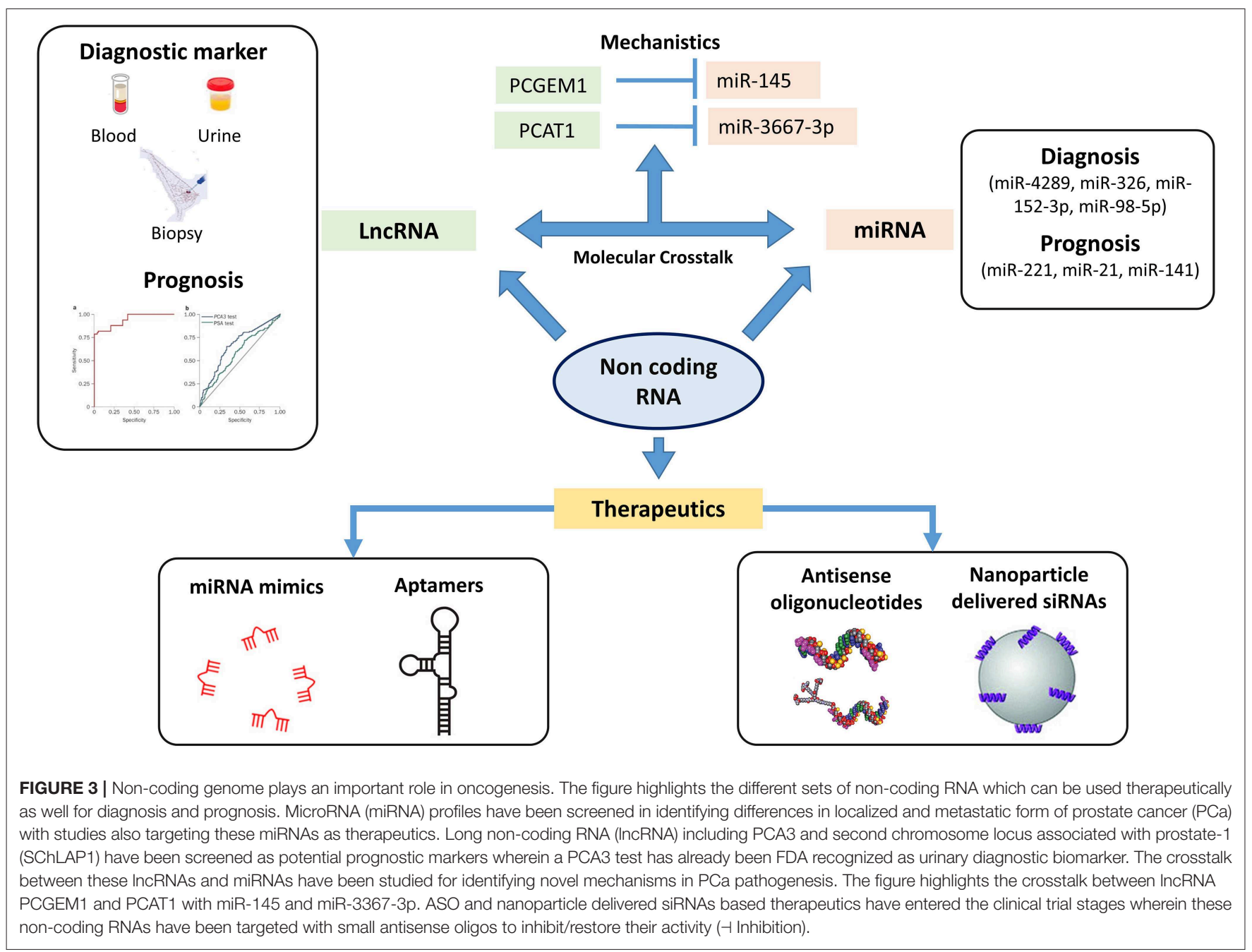

tested in clinics for several years along with RNA-based therapies. Eight of these therapies have reached phase III clinical trials until 2016 (109). miR-16 has been found to inhibit EMT by regulating $p-F A k$ and $p$-Akt expression, inhibiting the transcriptional efficiency of $N F-k B$ and Slug transcription factors (110). In one of the phase I trials, miR mimetics were used to restore miR-16 (TargomiRs) activity. In a study at Wisconsin Medical College, blood samples were collected at the time of ADT, posttreatment, and upon tumor progression to identify miRNAs and define changes in disease response (ClinicalTrials.gov Identifier: Nbib2366494). The study, once completed, aims to help in identifying specific miRNA changes at different levels of disease treatment for precise decision making affecting patient care. In a clinical phase II study, abiraterone, an androgen synthesis inhibitor, sensitivity to circulating miRNAs are being validated for prognosticating PCa progression (ClinicalTrials.gov Identifier: Nbib1503229). A research study conducted by Liu and group showed miR-34a via repressing CD44 reduced prostate tumor and metastasis in mice (111). This study was the basis of the first miRNA-based therapeutic trial, MXR34 (Nbib1829971), in men delivering the miRNA using liposome nanoparticles
(41). This study was conducted in liver cancer patients as the nanoparticles containing the RNA accumulated in liver tissues. These studies could be used to further change the delivery method for prostate-specific targeting. All these clinical studies on miRNA in PCa could lead to a panel of miRNA biomarkers in addition to imaging techniques for predicting disease stage and burden. If these stable miRNAs could predict the primary or metastatic stages of $\mathrm{PCa}$, it would lead to better treatment usage like chemotherapy and androgen-deprivation therapy altering the overall survival of patients.

CircRNAs were primarily studied in plants infected with viruses and subsequently confirmed in animal cells and fungal yeast $(112,113)$. These RNAs are stable and expressed in saliva, blood, and other body fluids $(114,115)$. However, these circRNAs have now been studied in PCa tumorigenesis and progressions and their mechanism investigated. To determine the potential of circRNAs as biomarkers, Xia et al. analyzed their expression profiles in prostate tumor and precancerous tissues (116). One thousand twenty-one differentially expressed circRNAs were found in the PCa tumor. The results found two circRNAs_circ_0057558 and circ_0062019-combined with 
PSA distinguished $\mathrm{PCa}$ and $\mathrm{BPH}$ patients with increased AUC, sensitivity, and specificity (0.938, 84.5 and $90.9 \%$, respectively) compared to PSA alone (AUC of serum PSA -0.854). Another circRNA, circRNA myosin light chain kinase was found to be upregulated in PCa tissues and cells, increasing cell proliferation, invasiveness, and migration (117). The levels of circRNA-MYK were found to be affected by miR-29a underlying the sponge mechanism of these RNAs. miR-29a expression was found to be lower in tumor samples compared to normal tissues, negatively correlating with the expression of circRNA-myosin light chain kinase. Recently, a study identified hsa_circ_0004870 to be associated with enzalutamide resistance in $\mathrm{PCa}$, highlighting critical roles of these RNAs in developing resistance in CRPC (118). Likewise, circ-102004 expression was also found to be higher in PCa tissues having an oncogenic role in stimulating cancer cell migration and invasion (119). Emerging interests in developing these circRNAs for diagnostic and therapeutic purposes have resulted in several databases being produced like CircBase, CIRCpedia, and CSCD to help understand the mechanism and potential of these RNAs (99). A study by Vo et al. discovered circAURKA as a potential marker for neuroendocrine PCa. RNA-seq on 144 localized prostate tumors by Chen et al. identified circCSNK1G3 interacting with miR-181 and promoting cell growth (120). Taken together, all these studies and the emerging circRNA databases have advanced the knowledge of exploring these RNAs as new targets. In addition, some of these studies also highlight their importance in developing novel biomarkers at different stages of PCa.

Recent studies have revealed lncRNAs like prostate cancer antigen 3 (PCA3), second chromosome locus associated with prostate-1 (SChLAP1), and PCa associated non-coding RNA transcripts (PCATs) as potential prognostic biomarkers. PCA3 is found to be upregulated in prostate tumors in comparison to non-tumor cells (121). PCA3 can be identified in urine, and the PROGENSA PCA3 test is the primary urine-based molecular diagnostic test sanctioned by the FDA (122). In addition, metaanalysis of several studies established the validity of urine PCA3 levels for PCa diagnosis, with a sensitivity of $62 \%$ and a selectivity of $75 \%$. In the receiver operating characteristic curve (ROC), this outcome interpreted as an AUC of 0.75, promoting PCA3 as a sensible marker for PCa diagnosis (123). Comparable outcomes were acquired in a subsequent independent metaanalysis wherein the sensitivity and selectivity for PCa diagnosis were 57 and $71 \%$, respectively; correspondingly, the AUC was 0.711 (124). As its plasma expression levels draw a parallel with tumor aggressiveness, as categorized by the Gleason score, circulating PCA3 can also reveal the aggressiveness of PCa (125). Although PCA3 improves the specificity for PCa detection in comparison to serum PSA, it is not sufficient to use it alone in making a decision for initial biopsy due to its lower sensitivity (125). Multiparametric magnetic resonance imaging (mpMRI) is a technique increasingly being used for prostate tumor detection and localization with high sensitivity and high-negative predictive value. Biomarkers like PCA3 in combination with mpMRI could predict high grade PCa more suitably. An observational study led by Hendriks and colleagues investigated association among a novel biomarker-based risk score (SelectMDx, MDxHealth, Irvine, CA), mpMRI outcomes, and biopsy Gleason score (126). The novel SelectMDx risk score surpassed PSA and PCA3 in the ROC curve analysis with an AUC of 0.83 vs. 0.66 and 0.65 . The study suggested using this novel risk score to identify PCa risk patients for advanced diagnostics, reducing overtreatment and avoidable diagnosis. Recently, Sanda et al. argued on collective urine based evaluation of PCA3 and TMPRSS:ERG fusion after DRE to improve specificity for aggressive PCa and for fending needless biopsies, which could prevent increased healthcare costs (127). Van Neste et al. evaluated a urine-based molecular marker risk score to aid in detection of high-grade PCa. This messenger RNA (mRNA) test combined molecular profiling data with clinical risk factors to precisely identify patients with high-grade PCa. mRNA expression levels of HOXC6 and DLX1 combined with $P S A$, age, DRE, prostate biopsy, and family history were used to detect high-grade $\mathrm{PCa}$ accurately with the researchers claiming that, clinically, it could reduce biopsies and overtreatment (128). Another group examined the ratio of Hepsin mRNA to PCA3 with serum PSA to improve prediction of PCa status. The results showed the ratio of Hepsin:PCA3 with serum PSA as a better predictor than PSA alone of PCa status and risk (129). These results are still not sufficient enough to be recommended as stand-alone tests for identifying PCa; however, these do provide a basis for adding other markers to improve patient diagnosis and selecting patients for biopsies and treatment.

Many lncRNAs also play crucial roles in cancer, from acting as oncogenes or tumor suppressors to regulating oncogenes and tumor suppressors at transcriptional and posttranscriptional levels (130). The idea of targeting lncRNA has been explored by researchers worldwide. In a study by Kanduri et al., the function of lncRNA, SCAT7, was blocked using ASO to reduce lung tumors in mice (131). These ASOs were observed to reduce lung tumors by $40-50 \%$ when injected twice a week and in 15 days. ARLNC1, an IncRNA, was found as a novel regulator of AR signaling, highlighting its potential as therapeutic target for advanced PCa (132). The application of ASOs has made possible of them being used to target the so-called "undruggable" genome like the lncRNA at the transcript level in PCa treatment. Understanding the molecular mechanism of lncRNA in PCa pathogenesis could yield a new "lncRNA therapy" being developed.

RNA interference (RNAi) using siRNA to treat disease have been the most effective method to date with the first ever drug approved by FDA treating hereditary transthyretin-mediated amyloidosis already in the market. This double-stranded RNA molecule could also become an effective cancer therapy. Various clinical trials are at an advanced stage using this molecule for treating cancer (133). siRNA-based approach has also gained momentum for treating PCa. Recently, nanoparticle-conjugated siRNA targeting PSMA showed significant inhibition of PCa tumor growth in vivo (134). Similar results were obtained in mice using an siRNA conjugated with squalene, a non-ionic lipid, targeting TMPRSS2-ERG junction oncogene (135). Other molecules like aptamers, which are single-stranded RNA or DNA oligonucleotides, have also been explored as agonists, antagonists, as well as effective drug conjugates (136). OX40, 
an agonistic aptamer targeting CD134 and 4-1BB, activated T cells and showed antitumor effects $(137,138)$. This aptamer targeting PSMA has also shown to reduce side effects. Additional research using this molecule has also led to developing an aptamer-antibody complex, called as oligobody, which reduced tumor burden in mice (139). Similar results using aptamer-drug conjugates were obtained in PCa cells, without causing harm to normal tissues (140). These kinds of studies indeed highlight the use of aptamer-based therapies as safe options to treat PCa.

All these types of ncRNAs do provide a novel "out-of-the-box" methods to target $\mathrm{PCa}$; however, precision medicine does need approaches for efficient delivery of these RNAs. The complex RNA-based therapeutics including miRNA mimics and siRNAs do need to overcome the problem of half-life and increased stability to be used in clinics. In addition, miRNAs and lncRNAs have been suggested to be useful biomarkers for monitoring treatment outcomes and responses, although all of them are still in preclinical phases. Only a few of them exist in body fluids, enabling a non-invasive liquid biopsy approach. The short ncRNAs like miRNA and siRNA have been advantageous for safe circulation and uptake, improving bioavailability, which has seen rapid development of clinical trials using these RNAs. Going forward, modifications including nanoparticles and alterations in ncRNA structure could help in overcoming toxicity and reducing off-target effects. Despite these challenges, ncRNAbased therapeutics are envisage to be a powerful treatment strategy for cancer treatment.

\section{LIQUID BIOPSY}

Tumor heterogeneity has made assessing variations in solid tumors extremely difficult, undermining effective treatment. Disadvantages of tissue biopsies include risk of infection and prolonged recovery time for patients, insufficient tissue for histological and molecular analysis of patients, and rebiopsy for additional genotyping and molecular profiling (141). PSA levels as an invasive test has failed to monitor disease burden and identify impact of therapies, reflecting its inefficiency as a dominant marker for PCa monitoring. Looking into the limitations of traditional biopsy, liquid biopsy gives an alternative approach as an advancing diagnostic tool. The limiting factors for improved treatment decisions-clonal variations and heterogeneity-are better evaluated while analyzing circulating components of blood. Furthermore, the genetic profile of patient tumor subclones is better reflected by liquid biopsy rather than tissue biopsies (142). The potential clinical utility of molecules identified in liquid biopsy includes cell free DNA (cfDNA), circulating tumor cells (CTCs), cell free proteins, exosomes, miRNAs, IncRNAs, mRNAs, and peptides (143). CTCs along with circulating tumor DNA (ctDNA) have been explored to ascertain alterations in the genome in PCa and trace the differential genomic landscape over a period of time (14).

With broader applications of next-generation sequencing technology, the use of ctDNA in screening genetic lesions have become highly specific and increasingly sensitive. From expediting early-stage detection to precisely determining tumor progression, prognosis, and assisting targeted therapy, the use of ctDNA in liquid biopsy represents a revolution in cancer diagnosis, prognosis, and treatment (142). New technologies including digital PCR-based methods, personalized analysis of rearranged beads, tagged amplicon deep sequencing, and untargeted ctDNA approaches have been developed to detect mutations and chromosomal rearrangements in ctDNA. The use of digital PCR yields detection of ctDNA in more than 75\% of advanced cancer patients, whereas in patients with localized tumors, the range is around $48-73 \%$ (144). The use of ctDNA offers a viable approach in clinical settings for patients with less pain and invasiveness compared to a tissue biopsy method. Recently, a team lead by Sonpavde et al. analyzed ctDNA from blood samples of 514 patients suffering from CRPC. The research found linkage between DNA changes and poor clinical outcomes in 163 patients, 46 of which were treated for CRPC before the study. A high number of genetic alterations were found in AR gene, similar to tumor tissue alterations, and were associated with poor treatment outcomes. The data also detected new $A R, M Y C$, and $B R C A$ alterations following therapy, which could be targeted using agents like immune checkpoint and PARP inhibitors (145).

A secondary approach in liquid biopsy of $\mathrm{PCa}$ patients is the examination of CTCs. These cells have their origin from the primary tumor or metastasis and can be detected in blood. Initially thought as a measure of disease aggressiveness, CTCs with emerging detection technologies have been studied and investigated for diagnosis and cancer management. The advantage of CTCs has been ease of isolation of pure tumor DNA and RNA from single cells for analyzing splice variants, which play a significant role in developing resistance to ADT in men suffering from PCa (146). The development of functional CTC studies has been a challenging task, as only a small number of CTCs can be salvaged from patient blood. To overcome this, new technologies are being developed for CTC culturing. Gao et al. derived $3 \mathrm{D}$ organ cultures from patients with advanced PCa, paving the way for the application of CTC cell lines in drug development and targeted therapy (147). Identifying variants of $\mathrm{AR}$ via liquid biopsy gives clinicians treating patients with enzalutamide or abiraterone based on resistance and sensitivity. For example, Borgmann et al. checked the potency of darolutamide, a chemically distinct drug compared to enzalutamide and other $\mathrm{AR}$ inhibitors. It was shown to have a higher binding affinity to AR, inhibiting cell growth and AR transcriptional activity in enzalutamide-resistant CRPC (148). Based on AR mutational status, probably in CTCs and its sensitivity to darolutamide, it can be used in precision oncology targeting mutated form of AR. CTCs have extensive heterogeneity, showing capacity to expand and form clusters that can traverse narrow capillaries and retain their properties upon reaching wider blood vessels (149). These clusters have been found to metastasize compared to single CTCs, ultimately decreasing overall survival of patients (150). Recently, Gkountela et al. profiled the DNA methylation landscape of single vs. clustered CTCs from breast cancer patients and mouse models to dig deeper into the biological features of CTC clusters (151). These CTC clusters shared several properties with stem cells including regulating self-renewal and proliferation. The study 
found hypermethylated sites for transcription factors associated with proliferation and stemness in CTC clusters. The researchers also identified $\mathrm{NA}+/ \mathrm{K}+$ ATPase inhibitors to be enabling in dissociating CTC clusters and remodeling DNA methylation and metastatic suppression. The phenotypic features of CTC clusters and an insight into their biology published in this study provide a rationale for applying compounds for treating breast cancer patients.

Currently, an FDA-approved CTC test by CELLSEARCH ${ }^{\circledR}$ system (Menarini Silicon Biosystems) is being used to detect CTCs in peripheral blood (152). The kit detects CTCs of epithelial origin in whole blood, which helps in monitoring patients with prostate, colorectal, or metastatic breast cancer. CELLSEARCH ${ }^{\circledR}$ CTC test has been argued to be used with other imaging and laboratory tests and physical examination for better prediction. This test relies on a single marker (EpCAM+) for CTC isolation, which has shown limitations. Another test by a German company, The Maintrac ${ }^{\circledR}$ CTC count test, measures cancer stem cells in blood. The CTC count indicates cancer aggressiveness and monitors treatment response. These tests, used every 3-6 months, is the best way to determine the risk of cancer spread and relapse, monitor treatment efficacy, and assess cancer aggressiveness. Many studies have proven the significance of Maintrac ${ }^{\circledR}$ diagnostics and are being employed in the case of almost all solid tumors (153-155). A group of Chinese researchers claimed to develop a new platform, subtraction enrichment and immunostaining-FISH to analyze CTCs for early screening of cancer in healthy people (156). They claimed the subtraction enrichment and immunostainingFISH platform developed had a higher CTC detection rated than the CELLSEARCH system. The subtraction enrichment of the platform used immunomagnetic particles conjugated with anti-CD45 antibody, which does not depend on EpCAM expression of CTCs that may decrease during EMT. The platform identified aneuploidy CTCs in addition to cytokeratin $+(\mathrm{CK}+)$ CTCs (157).

In summary, these technologies represent a potential tool for early-stage cancer screening as well as detecting aggressiveness and monitoring treatment efficacy relapse, providing a source for the use of liquid biopsy for precision medicine.

\section{SUMMARY}

The belief of precision oncology stems from developing effective therapeutic approaches for individual patients. Identification of new disease pathways and the development of pathway inhibitors have indeed contributed to the decline in PCa mortality in the last decades. Nonetheless, these inhibitors have, over a period of time, led to developing resistance in patients. Henceforth, improvement in developing novel therapeutics is the way forward for treating PCa. Therapeutic interventions in gene fusions targeting ERG and other ETS family members are an important opportunity to derive novel inhibitors that could substitute the current chemotherapies to treat resistant and metastatic disease. From using docetaxel and cabazitaxel in improving survival rate of PCa patients to developing new ASOs, precision medicine in cancer has taken big leap in this decade. A randomized trial of using Custirsen (OGX011), a second-generation ASO inhibiting clusterin, an upregulated protein, during chemotherapy, in combination with cabazitaxel and prednisone in CRPC patients, was conducted in Europe where patients have been recruited from eight countries to check for increased survival after the treatment (ClinicalTrials.gov Identifier: NCT01578655). The results exhibited no survival benefits to metastatic CRPC patients resulting in a failed trial. This kind of trials even after exploratory analysis in the in vitro and in vivo systems fails to deliver on the promise of a personalized approach to treatment. These trials are still far-fetched from having efficacy; however, use of biomarker-driven strategies and scrutinizing the phase I/II proof of concept studies is the way forward. A recent analysis showed $\sim 75 \%$ drugs developed accomplished regulatory authorization with this idea of biomarker-driven studies and positive POC in comparison to $15-30 \%$ with no putative biomarker and negative or no POCs (158).

The capacity of proteomics to examine and evaluate human disease has been fascinating, leading to new approaches being developed in this century. Proteogenomics is being discussed worldwide to solve problems in diseases, and this integration of proteomics and genomics presents an important opportunity for clinicians for early intervention and consistent monitoring (159). MS has already revolutionized the fields of microbiology and bacteriology in clinical settings; its full potential in the field of clinical oncology is widely being debated. The increased capacity of MS-based qualitative assays has increased specificity in diagnostics. In spite of all its advantages, drawbacks including data acquisition and reproducibility provide a concern for proteomics to be used in clinics. The answer to these questions could lie in using untargeted DIA having high multiplexing and comprehensive quantification capability of thousands of analytes with high specificity and selectivity. Developing a robust MS system with automatic quality control which can be operated by a non-expert could allow clinicians to accept this technology and gain acceptance in the community.

Genome editing has redefined the scientific field with potential ranging from basic sciences to precision therapy approaches. The genotype-phenotype relationship in human cells can be altered using this strategy where disease models have been tailored to edit genetic variations (160). Although genome editing has been a fancied approach for many scientists, arguments regarding its efficacy and the stress induced by it on whole-genome levels make this revolution a social stigma.

The field of oncology was modernized when the non-coding genome was found to affect disease at different levels. Studies indicated that non-coding RNA could serve in the diagnosis and prognosis of cancer (161). RNAi targeting by miRNA and lncRNA has led to success in laboratory settings with clinical trials with non-coding RNA therapies well underway. The year 2018 was a landmark year for RNAi with FDA approving Alnylam's ONPATTRO (Patisiran), the first drug to use this system to reduce transthyretin expression, which causes transthyretin-mediated amyloidosis in adults (162). Other RNAi therapeutic trials are underway at phase III stages for hemophilia, hypercholesterolemia, and acute hepatic porphyrias (163). These efforts may be defining in generating new treatment modules using RNAi technology to improve precision medicine 
of cancer patients. Liquid biopsy profiling has opened a new avenue compared to the traditional tissue biopsies in harvesting biomarkers like CTCs CtDNA from body fluids. This approach could help evaluate relapse in patients already undergoing treatment and monitor treatment response, guiding to a more personalized approach to cancer treatment.

This concept of precision medicine propelled by the increasing knowledge of genomics, proteomics, and development of new technologies is still in its infancy. The major challenge in developing an efficient therapy is the heterogeneous and multigenic nature of cancers. The results from various studies worldwide look promising for the development of precision medicine in PCa in the near future. However, there is still more

\section{REFERENCES}

1. Bray F, Ferlay J, Soerjomataram I, Siegel RL, Torre LA, Jemal A. Global cancer statistics 2018: GLOBOCAN estimates of incidence and mortality worldwide for 36 cancers in 185 countries. CA Cancer J Clin. (2018). 68:394-424. doi: $10.3322 /$ caac. 21492

2. Olivier C, Bernard M. Biomarkers of aggressiveness in prostate cancer. In: Spiess PE, editor. Prostate Cancer - Diagnostic and Therapeutic Advances. Rijeka: InTech (2011). p. 3-20. doi: 10.5772/25310

3. Prensner JR, Rubin MA, Wei JT, Chinnaiyan AM. Beyond PSA: the next generation of prostate cancer biomarkers. Sci Transl Med. (2012) 4:127rv123. doi: 10.1126/scitranslmed.3003180

4. Qu M, Ren SC, Sun YH. Current early diagnostic biomarkers of prostate cancer. Asian J Androl. (2014) 16:549-54. doi: 10.4103/1008-682X.129211

5. Martin RM, Donovan JL, Turner EL, Metcalfe C, Young GJ, Walsh EI, et al. Effect of a low-intensity PSA-based screening intervention on prostate cancer mortality: the CAP randomized clinical trial. JAMA. (2018) 319:883-95. doi: 10.1001/jama.2018.0154

6. Huggins C, Hodges CV. Studies on prostatic cancer. I. the effect of castration, of estrogen and of androgen injection on serum phosphatases in metastatic carcinoma of the prostate. Cancer Res. (1941) 1:293.

7. Hotte SJ, Saad F. Current management of castrate-resistant prostate cancer. Curr Oncol. (2010) 17:S72-9. doi: 10.3747/co.v17i0.718

8. Beer TM, Armstrong AJ, Rathkopf DE, Loriot Y, Sternberg CN, Higano CS, et al. Enzalutamide in metastatic prostate cancer before chemotherapy. $N$ Engl J Med. (2014) 371:424-33. doi: 10.1056/NEJMoa1405095

9. Smith MR, Saad F, Chowdhury S, Oudard S, Hadaschik BA, Graff JN, et al. Apalutamide treatment and metastasis-free survival in prostate cancer. $N$ Engl J Med. (2018) 378:1408-18. doi: 10.1056/NEJMoa1715546

10. Litwin MS, Tan HJ. The diagnosis and treatment of prostate cancer: a review. JAMA. (2017) 317:2532-42. doi: 10.1001/jama.2017.7248

11. Quinn DI, Sandler HM, Horvath LG, Goldkorn A, Eastham JA. The evolution of chemotherapy for the treatment of prostate cancer. Ann Oncol. (2017) 28:2658-69. doi: 10.1093/annonc/mdx348

12. Wang G, Zhao D, Spring DJ, Depinho RA. Genetics and biology of prostate cancer. Genes Dev. (2018) 32:1105-40. doi: 10.1101/gad.315739.118

13. Kantoff PW, Higano CS, Shore ND, Berger ER, Small EJ, Penson DF, et al. Sipuleucel-T immunotherapy for castration-resistant prostate cancer. $N$ Engl J Med. (2010) 363:411-22. doi: 10.1056/NEJMoa1001294

14. Mullane SA, Van Allen EM. Precision medicine for advanced prostate cancer. Curr Opin Urol. (2016) 26:231-9. doi: 10.1097/MOU.0000000000000278

15. Ramakrishnan Geethakumari P, Schiewer MJ, Knudsen KE, Kelly WK. PARP inhibitors in prostate cancer. Curr Treat Options Oncol. (2017) 18:37. doi: 10.1007/s11864-017-0480-2

16. Mateo J, Carreira S, Sandhu S, Miranda S, Mossop H, Perez-Lopez R, et al. DNA-repair defects and olaparib in metastatic prostate cancer. $\mathrm{N} \mathrm{Engl} \mathrm{J} \mathrm{Med.}$ (2015) 373:1697-708. doi: 10.1056/NEJMoa1506859

17. Modena A, Iacovelli R, Scarpa A, Brunelli M, Ciccarese C, Fantinel E, et al. Investigating BRCA mutations: a breakthrough in precision medicine to comprehend before precision treatment of $\mathrm{PCa}$ can reach the clinics.

\section{AUTHOR CONTRIBUTIONS}

All authors listed have made a substantial, direct and intellectual contribution to the work, and approved it for publication.

\section{FUNDING}

JB was supported by NHMRC Career Development Fellowship, SS by Advance Qld Fellowship and a John-Mills Young Investigator Award, and AM by QUTPRA scholarship. of castration-resistant prostate cancer. Targeted Oncol. (2016) 11:569-77. doi: 10.1007/s11523-016-0450-9

18. Myers CE, Feldman R, Abbott BL, Reddy SK, Castro M. Frequency of BRCA mutations and co-occurring alterations in prostate cancer. J Clin Oncol. (2016) 34:289. doi: 10.1200/jco.2016.34.2_suppl.289

19. Castro E, Goh C, Olmos D, Saunders E, Leongamornlert D, Tymrakiewicz $\mathrm{M}$, et al. Germline BRCA mutations are associated with higher risk of nodal involvement, distant metastasis, and poor survival outcomes in prostate cancer. J Clin Oncol. (2013) 31:1748-57. doi: 10.1200/JCO.2012. 43.1882

20. Castro E, Goh C, Leongamornlert D, Saunders E, Tymrakiewicz M, Dadaev $\mathrm{T}$, et al. Effect of BRCA mutations on metastatic relapse and cause-specific survival after radical treatment for localised prostate cancer. Eur Urol. (2015) 68:186-93. doi: 10.1016/j.eururo.2014.10.022

21. Liu Q, Tong D, Liu G, Yi Y, Xu J, Yang X, et al. A novel BRCA2 mutation in prostate cancer sensitive to combined radiotherapy and androgen deprivation therapy. Cancer Biol Ther. (2018) 19:669-75. doi: 10.1080/15384047.2018.1451278

22. Barbieri CE, Tomlins SA. The prostate cancer genome: perspectives and potential. Urol Oncol. (2014) 32:53.e15-22. doi: 10.1016/j.urolonc.2013.08.025

23. Kruglyak L, Nickerson DA. Variation is the spice of life. Nat Genet. (2001) 27:234-6. doi: 10.1038/85776

24. Zheng SL, Sun J, Wiklund F, Smith S, Stattin P, Li G, et al. Cumulative association of five genetic variants with prostate cancer. $N$ Engl J Med. (2008) 358:910-9. doi: 10.1056/NEJMoa075819

25. Duncan L, Shen H, Gelaye B, Meijsen J, Ressler K, Feldman M, et al. Analysis of polygenic risk score usage and performance in diverse human populations. Nat Commun. (2019) 10:3328. doi: 10.1038/s41467-019-11112-0

26. Torkamani A, Wineinger NE, Topol EJ. The personal and clinical utility of polygenic risk scores. Nat Rev Genet. (2018) 19:581-90. doi: 10.1038/s41576-018-0018-x

27. Aly $\mathrm{M}$, Wiklund $\mathrm{F}, \mathrm{Xu}$ J, Isaacs WB, Eklund $\mathrm{M}$, D'amato $\mathrm{M}$, et al. Polygenic risk score improves prostate cancer risk prediction: results from the Stockholm-1 cohort study. Eur Urol. (2011) 60:21-8. doi: 10.1016/j.eururo.2011.01.017

28. Dudbridge F. Power and predictive accuracy of polygenic risk scores. PLoS Genet. (2013) 9:e1003348. doi: 10.1371/journal.pgen.1003348

29. Eeles RA, Olama AA, Benlloch S, Saunders EJ, Leongamornlert DA, Tymrakiewicz M, et al. Identification of 23 new prostate cancer susceptibility loci using the iCOGS custom genotyping array. Nat Genet. (2013) 45:385-91, 391.e1-2. doi: $10.1038 / \mathrm{ng} .2560$

30. Chatterjee N, Shi J, Garcia-Closas M. Developing and evaluating polygenic risk prediction models for stratified disease prevention. Nat Rev Genet. (2016) 17:392-406. doi: 10.1038/nrg.2016.27

31. Seibert TM, Fan CC, Wang Y, Zuber V, Karunamuni R, Parsons JK, et al. Polygenic hazard score to guide screening for aggressive prostate cancer: development and validation in large scale cohorts. BMJ. (2018) 360:j5757. doi: $10.1136 /$ bmj.j5757 
32. Pashayan N, Duffy SW, Neal DE, Hamdy FC, Donovan JL, Martin RM, et al. Implications of polygenic risk-stratified screening for prostate cancer on overdiagnosis. Genet Med. (2015) 17:789-95. doi: 10.1038/gim.2014.192

33. Eeles R, Goh C, Castro E, Bancroft E, Guy M, Al Olama AA, et al. The genetic epidemiology of prostate cancer and its clinical implications. Nat Rev Urol. (2014) 11:18-31. doi: 10.1038/nrurol.2013.266

34. Tosoian JJ, Trock BJ, Landis P, Feng Z, Epstein JI, Partin AW, et al. Active surveillance program for prostate cancer: an update of the Johns Hopkins experience. J Clin Oncol. (2011) 29:2185-90. doi: 10.1200/JCO.2010.32.8112

35. Schumacher FR, Al Olama AA, Berndt SI, Benlloch S, Ahmed M, Saunders EJ, et al. Association analyses of more than 140,000 men identify 63 new prostate cancer susceptibility loci. Nat Genet. (2018) 50:928-36. doi: 10.1038/s41588-018-0142-8

36. Lecarpentier J, Silvestri V, Kuchenbaecker KB, Barrowdale D, Dennis J, Mcguffog L, et al. Prediction of breast and prostate cancer risks in male BRCA1 and BRCA2 mutation carriers using polygenic risk scores. J Clin Oncol. (2017) 35:2240-50. doi: 10.1200/JCO.2016.69.4935

37. Wray NR, Yang J, Hayes BJ, Price AL, Goddard ME, Visscher PM. Pitfalls of predicting complex traits from SNPs. Nat Rev Genet. (2013) 14:507-15. doi: $10.1038 / \mathrm{nrg} 3457$

38. Martin AR, Gignoux CR, Walters RK, Wojcik GL, Neale BM, Gravel $\mathrm{S}$, et al. Human demographic history impacts genetic risk prediction across diverse populations. Am J Hum Genet. (2017) 100:635-49. doi: 10.1016/j.ajhg.2017.03.004

39. Edwards PA. Fusion genes and chromosome translocations in the common epithelial cancers. J Pathol. (2010) 220:244-54. doi: 10.1002/path.2632

40. Kumar-Sinha C, Tomlins SA, Chinnaiyan AM. Recurrent gene fusions in prostate cancer. Nat Rev Cancer. (2008) 8:497-511. doi: 10.1038/nrc2402

41. Smits M, Mehra N, Sedelaar M, Gerritsen W, Schalken JA. Molecular biomarkers to guide precision medicine in localized prostate cancer. Exp Rev Mol Diagn. (2017) 17:791-804. doi: 10.1080/14737159.2017.1345627

42. Tomlins SA, Alshalalfa M, Davicioni E, Erho N, Yousefi K, Zhao S, et al. Characterization of 1577 primary prostate cancers reveals novel biological and clinicopathologic insights into molecular subtypes. Eur Urol. (2015) 68:555-67. doi: 10.1016/j.eururo.2015.04.033

43. Torres A, Alshalalfa M, Tomlins SA, Erho N, Gibb EA, Chelliserry J, et al. Comprehensive determination of prostate tumor ETS gene status in clinical samples using the CLIA decipher assay. J Mol Diagn. (2017) 19:475-84. doi: 10.1016/j.jmoldx.2017.01.007

44. Johnson MH, Ross AE, Alshalalfa M, Erho N, Yousefi K, Glavaris S, et al. SPINK1 defines a molecular subtype of prostate cancer in men with more rapid progression in an at risk, natural history radical prostatectomy cohort. J Urol. (2016) 196:1436-44. doi: 10.1016/j.juro.2016.05.092

45. Gasi Tandefelt D, Boormans J, Hermans K, Trapman J. ETS fusion genes in prostate cancer. Endocr Relat Cancer. (2014) 21:R143-52. doi: 10.1530/ERC-13-0390

46. Adamo P, Ladomery MR. The oncogene ERG: a key factor in prostate cancer. Oncogene. (2016) 35:403-14. doi: 10.1038/onc.2015.109

47. Zhao J, Chen Q, Wu J, Han P, Song X. GFusion: an effective algorithm to identify fusion genes from cancer RNA-Seq data. Sci Rep. (2017) 7:6880. doi: 10.1038/s41598-017-07070-6

48. Sanguedolce F, Cormio A, Brunelli M, D’amuri A, Carrieri G, Bufo P, et al. Urine TMPRSS2: ERG fusion transcript as a biomarker for prostate cancer: literature review. Clin Genitourin Cancer. (2016) 14:117-21. doi: 10.1016/j.clgc.2015.12.001

49. Tomlins SA, Day JR, Lonigro RJ, Hovelson DH, Siddiqui J, Kunju LP, et al. Urine TMPRSS2:ERG Plus PCA3 for individualized prostate cancer risk assessment. Eur Urol. (2016) 70:45-53. doi: 10.1016/j.eururo.2015.04.039

50. Mounir Z, Lin F, Lin VG, Korn JM, Yu Y, Valdez R, et al. TMPRSS2:ERG blocks neuroendocrine and luminal cell differentiation to maintain prostate cancer proliferation. Oncogene. (2015) 34:3815-25. doi: 10.1038/onc.2014.308

51. Feng FY, Brenner JC, Hussain M, Chinnaiyan AM. Molecular pathways: targeting ETS gene fusions in cancer. Clin Cancer Res. (2014) 20:4442-8. doi: 10.1158/1078-0432.CCR-13-0275

52. Hussain M, Daignault-Newton S, Twardowski PW, Albany C, Stein MN, Kunju LP, et al. Targeting androgen receptor and DNA repair in metastatic castration-resistant prostate cancer: results from NCI 9012. J Clin Oncol. (2018) 36:991-9. doi: 10.1200/JCO.2017.75.7310

53. Bradley D, Rathkopf D, Dunn R, Stadler WM, Liu G, Smith DC, et al. Vorinostat in advanced prostate cancer patients progressing on prior chemotherapy (National Cancer Institute Trial 6862): trial results and interleukin-6 analysis: a study by the Department of Defense Prostate Cancer Clinical Trial Consortium and University of Chicago Phase 2 Consortium. Cancer. (2009) 115:5541-9. doi: 10.1002/cncr.24597

54. Molife LR, Attard G, Fong PC, Karavasilis V, Reid AH, Patterson S, et al. Phase II, two-stage, single-arm trial of the histone deacetylase inhibitor (HDACi) romidepsin in metastatic castration-resistant prostate cancer (CRPC). Ann Oncol. (2010) 21:109-13. doi: 10.1093/annonc/mdp270

55. Federman N, Meyers PA, Daw NC, Toretsky J, Bradley Breitmeyer J, Singh AS, et al. TK216 in Patients With Relapsed or Refractory Ewing Sarcoma. Bethesda, MD: National Library of Medicine (US) (2016). Available online at: https://clinicaltrials.gov/ct2/show/study/NCT02657005

56. Butler MS, Roshan-Moniri M, Hsing M, Lau D, Kim A, Yen P, et al. Discovery and characterization of small molecules targeting the DNAbinding ETS domain of ERG in prostate cancer. Oncotarget. (2017) 8:4243854. doi: 10.18632/oncotarget.17124

57. Wang X, Qiao Y, Asangani IA, Ateeq B, Poliakov A, Cieslik M, et al. Development of peptidomimetic inhibitors of the ERG gene fusion product in prostate cancer. Cancer Cell. (2017) 31:532-48.e537. doi: 10.1016/j.ccell.2017.02.017

58. Luo J-H, Liu S, Zuo Z-H, Chen R, Tseng GC, Yu YP. Discovery and classification of fusion transcripts in prostate cancer and normal prostate tissue. Am J Pathol. (2015) 185:1834-45. doi: 10.1016/j.ajpath.2015.03.008

59. Zhao S, Løvf M, Carm KT, Bakken AC, Hoff AM, Skotheim RI. Novel transcription-induced fusion RNAs in prostate cancer. Oncotarget. (2017) 8:49133-43. doi: 10.18632/oncotarget.17099

60. Qin F, Zhang Y, Liu J, Li H. SLC45A3-ELK4 functions as a long non-coding chimeric RNA. Cancer Lett. (2017) 404:53-61. doi: 10.1016/j.canlet.2017.07.007

61. Lai J, An J, Seim I, Walpole C, Hoffman A, Moya L, et al. Fusion transcript loci share many genomic features with non-fusion loci. BMC Genomics. (2015) 16:1021. doi: 10.1186/s12864-015-2235-4

62. Geybels MS, Fang M, Wright JL, Qu X, Bibikova M, Klotzle B, et al. PTEN loss is associated with prostate cancer recurrence and alterations in tumor DNA methylation profiles. Oncotarget. (2017) 8:84338-48. doi: 10.18632/oncotarget.20940

63. Carver BS, Tran J, Gopalan A, Chen Z, Shaikh S, Carracedo A, et al. Aberrant ERG expression cooperates with loss of PTEN to promote cancer progression in the prostate. Nat Genet. (2009) 41:619-24. doi: 10.1038/ng.370

64. Jamaspishvili T, Berman DM, Ross AE, Scher HI, De Marzo AM, Squire JA, et al. Clinical implications of PTEN loss in prostate cancer. Nat Rev Urol. (2018) 15:222-34. doi: 10.1038/nrurol.2018.9

65. Mateo J, Ganji G, Lemech C, Burris HA, Han SW, Swales K, et al. A first-timein-human study of GSK2636771, a phosphoinositide 3 kinase beta-selective inhibitor, in patients with advanced solid tumors. Clin Cancer Res. (2017) 23:5981-92. doi: 10.1158/1078-0432.CCR-17-0725

66. Dallas DC, Guerrero A, Parker EA, Robinson RC, Gan J, German JB, et al. Current peptidomics: applications, purification, identification, quantification, and functional analysis. Proteomics. (2015) 15:1026-38. doi: 10.1002/pmic.201400310

67. Loeb S, Catalona WJ. The Prostate Health Index: a new test for the detection of prostate cancer. Ther Adv Urol. (2014) 6:74-7. doi: $10.1177 / 1756287213513488$

68. Kami K, Fujimori T, Sato H, Sato M, Yamamoto H, Ohashi Y, et al. Metabolomic profiling of lung and prostate tumor tissues by capillary electrophoresis time-of-flight mass spectrometry. Metabolomics. (2013) 9:444-53. doi: 10.1007/s11306-012-0452-2

69. Partin AW, Van Neste L, Klein EA, Marks LS, Gee JR, Troyer DA, et al. Clinical validation of an epigenetic assay to predict negative histopathological results in repeat prostate biopsies. J Urol. (2014) 192:10817. doi: 10.1016/j.juro.2014.04.013

70. Stewart GD, Van Neste L, Delvenne P, Delree P, Delga A, Mcneill SA, et al. Clinical utility of an epigenetic assay to detect occult prostate cancer in 
histopathologically negative biopsies: results of the MATLOC study. J Urol. (2013) 189:1110-6. doi: 10.1016/j.juro.2012.08.219

71. Shipitsin M, Small C, Choudhury S, Giladi E, Friedlander S, Nardone J, et al. Identification of proteomic biomarkers predicting prostate cancer aggressiveness and lethality despite biopsy-sampling error. $\mathrm{Br} J$ Cancer. (2014) 111:1201-12. doi: 10.1038/bjc.2014.396

72. Blume-Jensen P, Berman DM, Rimm DL, Shipitsin M, Putzi M, Nifong TP, et al. Development and clinical validation of an in situ biopsy-based multimarker assay for risk stratification in prostate cancer. Clin Cancer Res. (2015) 21:2591-600. doi: 10.1158/1078-0432.CCR-14-2603

73. Duijvesz D, Burnum-Johnson KE, Gritsenko MA, Hoogland AM, Vredenbregt-Van Den Berg MS, Willemsen R, et al. Proteomic profiling of exosomes leads to the identification of novel biomarkers for prostate cancer. PLoS ONE. (2013) 8:e82589. doi: 10.1371/journal.pone.0082589

74. Flatley B, Wilmott KG, Malone P, Cramer R. MALDI MS profiling of postDRE urine samples highlights the potential of beta-microseminoprotein as a marker for prostatic diseases. Prostate. (2014) 74:103-11. doi: $10.1002 /$ pros. 22736

75. Gronberg H, Adolfsson J, Aly M, Nordstrom T, Wiklund P, Brandberg Y, et al. Prostate cancer screening in men aged 50-69 years (STHLM3): a prospective population-based diagnostic study. Lancet Oncol. (2015) 16:1667-76. doi: 10.1016/S1470-2045(15)00361-7

76. Ström P, Nordström T, Aly M, Egevad L, Grönberg H, Eklund M. The Stockholm-3 model for prostate cancer detection: algorithm update, biomarker contribution, and reflex test potential. Eur Urol. (2018) 74:204-10. doi: 10.1016/j.eururo.2017.12.028

77. Möller A, Olsson H, Grönberg H, Eklund M, Aly M, Nordström T. The Stockholm3 blood-test predicts clinically-significant cancer on biopsy: independent validation in a multi-center community cohort. Prostate Cancer Prostatic Dis. (2019) 22:137-42. doi: 10.1038/s41391-018-0082-5

78. Heger Z, Michalek P, Guran R, Cernei N, Duskova K, Vesely S, et al. Differences in urinary proteins related to surgical margin status after radical prostatectomy. Oncol Rep. (2015) 34:3247-55. doi: 10.3892/or.2015.4322

79. Davalieva K, Kiprijanovska S, Komina S, Petrusevska G, Zografska NC, Polenakovic M. Proteomics analysis of urine reveals acute phase response proteins as candidate diagnostic biomarkers for prostate cancer. Proteome Sci. (2015) 13:2. doi: 10.1186/s12953-014-0059-9

80. Zhang M, Chen L, Yuan Z, Yang Z, Li Y, Shan L, et al. Combined serum and EPS-urine proteomic analysis using iTRAQ technology for discovery of potential prostate cancer biomarkers. Discov Med. (2016) 22:281-95.

81. Fujita K, Kume H, Matsuzaki K, Kawashima A, Ujike T, Nagahara A, et al. Proteomic analysis of urinary extracellular vesicles from high Gleason score prostate cancer. Sci Rep. (2017) 7:42961. doi: 10.1038/srep42961

82. Katafigioti A, Katafigiotis I, Sfoungaristos S, Alamanis C, Stravodimos $\mathrm{K}$, Anastasiou I, et al. In the search of novel urine biomarkers for the early diagnosis of prostate cancer. Intracellular or secreted proteins as the target group? Where and how to search for possible biomarkers useful in the everyday clinical practice. Arch Ital Urol Androl. (2016) 88:195-200. doi: 10.4081/aiua.2016.3.195

83. Wang L, Skotland T, Berge V, Sandvig K, Llorente A. Exosomal proteins as prostate cancer biomarkers in urine: from mass spectrometry discovery to immunoassay-based validation. Eur J Pharm Sci. (2017) 98:80-5. doi: 10.1016/j.ejps.2016.09.023

84. Adeola HA, Calder B, Soares NC, Kaestner L, Blackburn JM, Zerbini LF. In silico verification and parallel reaction monitoring prevalidation of potential prostate cancer biomarkers. Future Oncol. (2016) 12:43-57. doi: $10.2217 /$ fon. 15.296

85. Arora A, Patil V, Kundu P, Kondaiah P, Hegde AS, Arivazhagan A, et al. Serum biomarkers identification by iTRAQ and verification by MRM: S100A8/S100A9 levels predict tumor-stroma involvement and prognosis in Glioblastoma. Sci Rep. (2019) 9:2749. doi: 10.1038/s41598-019-39067-8

86. Wu D, Ni J, Beretov J, Cozzi P, Willcox M, Wasinger V, et al. Urinary biomarkers in prostate cancer detection and monitoring progression. Crit Rev Oncol Hematol. (2017) 118:15-26. doi: 10.1016/j.critrevonc.2017.08.002

87. Anjo SI, Santa C, Manadas B. SWATH-MS as a tool for biomarker discovery: from basic research to clinical applications. Proteomics. (2017). 17:1600278. doi: $10.1002 /$ pmic. 201600278
88. Latonen L, Afyounian E, Jylhä A, Nättinen J, Aapola U, Annala M, et al. Integrative proteomics in prostate cancer uncovers robustness against genomic and transcriptomic aberrations during disease progression. Nat Commun. (2018) 9:1176. doi: 10.1038/s41467-018-03573-6

89. Fortin T, Salvador A, Charrier JP, Lenz C, Lacoux X, Morla A, et al. Clinical quantitation of prostate-specific antigen biomarker in the low nanogram/milliliter range by conventional bore liquid chromatographytandem mass spectrometry (multiple reaction monitoring) coupling and correlation with ELISA tests. Mol Cell Proteomics MCP. (2009) 8:1006-15. doi: 10.1074/mcp.M800238-MCP200

90. Ho TT, Zhou N, Huang J, Koirala P, Xu M, Fung R, et al. Targeting noncoding RNAs with the CRISPR/Cas9 system in human cell lines. Nucleic Acids Res. (2015) 43:e17. doi: 10.1093/nar/gku1198

91. Smith KO, Galloway KS, Kennell WL, Ogilvie KK, Radatus BK. A new nucleoside analog, 9-[[2-hydroxy-1(hydroxymethyl)ethoxyl]methyl]guanine, highly active in vitro against herpes simplex virus types 1 and 2. Antimicrobial Agents Chemother. (1982) 22:55-61. doi: 10.1128/AAC.22.1.55

92. Chen ZH, Yu YP, Zuo ZH, Nelson JB, Michalopoulos GK, Monga S, et al. Targeting genomic rearrangements in tumor cells through Cas9mediated insertion of a suicide gene. Nat Biotechnol. (2017) 35:543-50. doi: $10.1038 /$ nbt. 3843

93. Liu J-J, Orlova N, Oakes BL, Ma E, Spinner HB, Baney KLM, et al. CasX enzymes comprise a distinct family of RNA-guided genome editors. Nature. (2019) 566:218-23. doi: 10.1038/s41586-019-0908-x

94. Ye R, Pi M, Cox JV, Nishimoto SK, Quarles LD. CRISPR/Cas9 targeting of GPRC6A suppresses prostate cancer tumorigenesis in a human xenograft model. J Exp Clin Cancer Res. (2017) 36:90. doi: 10.1186/s13046-017-0561-x

95. Zhen S, Takahashi Y, Narita S, Yang YC, Li X. Targeted delivery of CRISPR/Cas9 to prostate cancer by modified gRNA using a flexible aptamer-cationic liposome. Oncotarget. (2017) 8:9375-87. doi: $10.18632 /$ oncotarget.14072

96. Brosnan CA, Voinnet O. The long and the short of noncoding RNAs. Curr Opin Cell Biol. (2009) 21:416-25. doi: 10.1016/j.ceb.2009.04.001

97. Gibb EA, Brown CJ, Lam WL. The functional role of long noncoding RNA in human carcinomas. Mol Cancer. (2011) 10:38. doi: 10.1186/1476-4598-10-38

98. Derrien T, Johnson R, Bussotti G, Tanzer A, Djebali S, Tilgner H, et al. The GENCODE v7 catalog of human long noncoding RNAs: analysis of their gene structure, evolution, and expression. Genome Res. (2012) 22:1775-89. doi: $10.1101 /$ gr.132159.111

99. Vo JN, Cieslik M, Zhang Y, Shukla S, Xiao L, Zhang Y, et al. The landscape of circular RNA in cancer. Cell. (2019) 176:869-81.e813. doi: 10.1016/j.cell.2018.12.021

100. Kumar B, Lupold SE. MicroRNA expression and function in prostate cancer: a review of current knowledge and opportunities for discovery. Asian $J$ Androl. (2016) 18:559-67. doi: 10.4103/1008-682X.177839

101. Lichner Z, Ding Q, Samaan S, Saleh C, Nasser A, Al-Haddad S, et al. miRNAs dysregulated in association with Gleason grade regulate extracellular matrix, cytoskeleton and androgen receptor pathways. J Pathol. (2015) 237:226-37. doi: 10.1002/path. 4568

102. Lichner Z, Fendler A, Saleh C, Nasser AN, Boles D, Al-Haddad $\mathrm{S}$, et al. MicroRNA signature helps distinguish early from late biochemical failure in prostate cancer. Clin Chem. (2013) 59:1595-603. doi: 10.1373/clinchem.2013.205450

103. Porzycki P, Ciszkowicz E, Semik M, Tyrka M. Combination of three miRNA (miR-141, miR-21, and miR-375) as potential diagnostic tool for prostate cancer recognition. Int Urol Nephrol. (2018) 50:1619-26. doi: 10.1007/s11255-018-1938-2

104. Matin F, Jeet V, Clements JA, Yousef GM, Batra J. MicroRNA theranostics in prostate cancer precision medicine. Clin Chem. (2016) 62:1318-33. doi: 10.1373 /clinchem. 2015.242800

105. Li W, Ruan K. MicroRNA detection by microarray. Anal Bioanal Chem. (2009) 394:1117-24. doi: 10.1007/s00216-008-2570-2

106. Moldovan L, Batte KE, Trgovcich J, Wisler J, Marsh CB, Piper M. Methodological challenges in utilizing miRNAs as circulating biomarkers. $J$ Cell Mol Med. (2014) 18:371-90. doi: 10.1111/jcmm.12236 
107. Zhang K, Wang K, Zhu X, Xie M. A one-pot strategy for the sensitive detection of miRNA by catalyst-oligomer-mediated enzymatic amplification-based fluorescence biosensor. Sensors Actuators B Chem. (2016) 223:586-90. doi: 10.1016/j.snb.2015.09.153

108. Di Leva G, Garofalo M, Croce CM. MicroRNAs in cancer. Annu Rev Pathol. (2014) 9:287-314. doi: 10.1146/annurev-pathol-012513-104715

109. Smith B, Agarwal P, Bhowmick NA. MicroRNA applications for prostate, ovarian and breast cancer in the era of precision medicine. Endocr Relat Cancer. (2017) 24:R157-72. doi: 10.1530/ERC-16-0525

110. Wang Q, Li X, Zhu Y, Yang P. MicroRNA-16 suppresses epithelialmesenchymal transitionrelated gene expression in human glioma. Mol Med Rep. (2014) 10:3310-4. doi: 10.3892/mmr.2014.2583

111. Liu C, Kelnar K, Liu B, Chen X, Calhoun-Davis T, Li H, et al. The microRNA miR-34a inhibits prostate cancer stem cells and metastasis by directly repressing CD44. Nat Med. (2011) 17:211-5. doi: 10.1038/nm.2284

112. Hsu MT, Coca-Prados M. Electron microscopic evidence for the circular form of RNA in the cytoplasm of eukaryotic cells. Nature. (1979) 280:339-40. doi: $10.1038 / 280339 \mathrm{a} 0$

113. Arnberg AC, Van Ommen GJ, Grivell LA, Van Bruggen EF, Borst P. Some yeast mitochondrial RNAs are circular. Cell. (1980) 19:313-9. doi: 10.1016/0092-8674(80)90505-X

114. Romero Otero J, Garcia Gomez B, Campos Juanatey F, Touijer KA. Prostate cancer biomarkers: an update. Urol Oncol. (2014) 32:252-60. doi: 10.1016/j.urolonc.2013.09.017

115. Zhang XO, Wang HB, Zhang Y, Lu X, Chen LL, Yang L. Complementary sequence-mediated exon circularization. Cell. (2014) 159:134-47. doi: 10.1016/j.cell.2014.09.001

116. Xia Q, Ding T, Zhang G, Li Z, Zeng L, Zhu Y, et al. Circular RNA expression profiling identifies prostate cancer- specific circRNAs in prostate cancer. Cell Physiol Biochem. (2018) 50:1903-15. doi: 10.1159/000494870

117. Dai Y, Li D, Chen X, Tan X, Gu J, Chen M, et al. Circular RNA Myosin Light Chain Kinase (MYLK) promotes prostate cancer progression through modulating Mir-29a expression. Med Sci monit. (2018) 24:3462-71. doi: 10.12659/MSM.908009

118. Greene J, Baird A-M, Casey O, Brady L, Blackshields G, Lim M, et al. Circular RNAs are differentially expressed in prostate cancer and are potentially associated with resistance to enzalutamide. Sci Rep. (2019) 9:10739. doi: 10.1038/s41598-019-47189-2

119. Si-Tu J, Cai Y, Feng T, Yang D, Yuan S, Yang X, et al. Upregulated circular RNA circ-102004 that promotes cell proliferation in prostate cancer. Int J Biol Macromol. (2019) 122:1235-43. doi: 10.1016/j.ijbiomac.2018.09.076

120. Chen S, Huang V, Xu X, Livingstone J, Soares F, Jeon J, et al. Widespread and functional RNA circularization in localized prostate cancer. Cell. (2019) 176:831-843.e822. doi: 10.1016/j.cell.2019.01.025

121. Salagierski M, Schalken JA. PCA3 and TMPRSS2-ERG: promising biomarkers in prostate cancer diagnosis. Cancers. (2010) 2:1432-40. doi: 10.3390/cancers2031432

122. Lee GL, Dobi A, Srivastava S. Prostate cancer: diagnostic performance of the PCA3 urine test. Nat Rev Urol. (2011) 8:123-4. doi: 10.1038/nrurol.2011.10

123. Xue WJ, Ying XL, Jiang JH, Xu YH. Prostate cancer antigen 3 as a biomarker in the urine for prostate cancer diagnosis: a meta-analysis. J Cancer Res Ther. (2014) 10(Suppl):C218-21. doi: 10.4103/0973-1482.145881

124. Hu B, Yang H, Yang H. Diagnostic value of urine prostate cancer antigen 3 test using a cutoff value of $35 \mathrm{mug} / \mathrm{L}$ in patients with prostate cancer. Tumour Biol. (2014) 35:8573-80. doi: 10.1007/s13277-014-2109-4

125. Merola R, Tomao L, Antenucci A, Sperduti I, Sentinelli S, Masi S, et al. PCA3 in prostate cancer and tumor aggressiveness detection on 407 highrisk patients: a National Cancer Institute experience. J Exp Clin Cancer Res. (2015) 34:15. doi: 10.1186/s13046-015-0127-8

126. Hendriks RJ, Van Der Leest MMG, Dijkstra S, Barentsz JO, Van Criekinge W, Hulsbergen-Van De Kaa CA, et al. A urinary biomarker-based risk score correlates with multiparametric MRI for prostate cancer detection. Prostate. (2017) 77:1401-7. doi: 10.1002/pros.23401

127. Sanda MG, Feng Z, Howard DH, Tomlins SA, Sokoll LJ, Chan DW, et al. Association between combined TMPRSS2:ERG and PCA3 RNA urinary testing and detection of aggressive prostate cancer. JAMA Oncol. (2017) 3:1085-93. doi: 10.1001/jamaoncol.2017.0177
128. Van Neste L, Hendriks RJ, Dijkstra S, Trooskens G, Cornel EB, Jannink SA, et al. Detection of high-grade prostate cancer using a urinary molecular biomarker-based risk score. Eur Urol. (2016) 70:740-8. doi: 10.1016/j.eururo.2016.04.012

129. Roberts MJ, Chow CW, Schirra HJ, Richards R, Buck M, Selth LA, et al. Diagnostic performance of expression of PCA3, Hepsin and miR biomarkers inejaculate in combination with serum PSA for the detection of prostate cancer. Prostate. (2015) 75:539-49. doi: 10.1002/pros.22942

130. Arun G, Diermeier SD, Spector DL. Therapeutic targeting of long non-coding RNAs in cancer. Trends Mol Med. (2018) 24:257-77. doi: 10.1016/j.molmed.2018.01.001

131. Ali MM, Akhade VS, Kosalai ST, Subhash S, Statello L, MeryetFiguiere M, et al. PAN-cancer analysis of S-phase enriched lncRNAs identifies oncogenic drivers and biomarkers. Nat Commun. (2018) 9:883. doi: 10.1038/s41467-018-03265-1

132. Zhang Y, Pitchiaya S, Cieślik M, Niknafs YS, Tien JCY, Hosono Y, et al. Analysis of the androgen receptor-regulated lncRNA landscape identifies a role for ARLNC1 in prostate cancer progression. Nat Genet. (2018) 50:81424. doi: 10.1158/1538-7445.AM2018-2458

133. Mahmoodi Chalbatani G, Dana H, Gharagouzloo E, Grijalvo S, Eritja $\mathrm{R}$, Logsdon CD, et al. Small interfering RNAs (siRNAs) in cancer therapy: a nano-based approach. Int J Nanomed. (2019) 14:3111-28. doi: 10.2147/IJN.S200253

134. Xu X, Wu J, Liu Y, Saw PE, Tao W, Yu M, et al. Multifunctional envelopetype siRNA delivery nanoparticle platform for prostate cancer therapy. ACS Nano. (2017) 11:2618-27. doi: 10.1021/acsnano.6b07195

135. Urbinati G, Ali HM, Rousseau Q, Chapuis H, Desmaële D, Couvreur $P$, et al. Antineoplastic effects of siRNA against TMPRSS2-ERG junction oncogene in prostate cancer. PLoS ONE. (2015) 10:e0125277. doi: 10.1371/journal.pone.0125277

136. Kim M, Kim D-M, Kim K-S, Jung W, Kim D-E. Applications of cancer cell-specific aptamers in targeted delivery of anticancer therapeutic agents. Molecules. (2018) 23:830. doi: 10.3390/molecules23040830

137. Dollins CM, Nair S, Boczkowski D, Lee J, Layzer JM, Gilboa E, et al. Assembling OX40 aptamers on a molecular scaffold to create a receptor-activating aptamer. Chem Biol. (2008) 15:675-82. doi: 10.1016/j.chembiol.2008.05.016

138. Pastor F, Kolonias D, Mcnamara JO II, Gilboa E. Targeting 4-1BB costimulation to disseminated tumor lesions with bi-specific oligonucleotide aptamers. Mol Ther. (2011) 19:1878-86. doi: 10.1038/mt.2011.145

139. Heo K, Min SW, Sung HJ, Kim HG, Kim HJ, Kim YH, et al. An aptamer-antibody complex (oligobody) as a novel delivery platform for targeted cancer therapies. J Control Release. (2016) 229:1-9. doi: 10.1016/j.jconrel.2016.03.006

140. Powell Gray B, Kelly L, Ahrens DP, Barry AP, Kratschmer C, Levy M, et al. Tunable cytotoxic aptamer-drug conjugates for the treatment of prostate cancer. Proc Natl Acad Sci. (2018) 115:4761-6. doi: 10.1073/pnas.1717705115

141. Ilié M, Hofman P. Pros: can tissue biopsy be replaced by liquid biopsy? Transl Lung Cancer Res. (2016) 5:420-3. doi: 10.21037/tlcr.2016.08.06

142. Cheng F, Su L, Qian C. Circulating tumor DNA: a promising biomarker in the liquid biopsy of cancer. Oncotarget. (2016) 7:48832-41. doi: 10.18632/oncotarget.9453

143. Di Meo A, Bartlett J, Cheng Y, Pasic MD, Yousef GM. Liquid biopsy: a step forward towards precision medicine in urologic malignancies. Mol Cancer. (2017) 16:80. doi: 10.1186/s12943-017-0644-5

144. Bettegowda C, Sausen M, Leary RJ, Kinde I, Wang Y, Agrawal $\mathrm{N}$, et al. Detection of circulating tumor DNA in early- and latestage human malignancies. Sci Transl Med. (2014) 6:224ra224. doi: 10.1126/scitranslmed.3007094

145. Sonpavde G, Agarwal N, Pond GR, Nagy RJ, Nussenzveig RH, Hahn AW, et al. Circulating tumor DNA alterations in patients with metastatic castration-resistant prostate cancer. Cancer. (2019) 125:1459-69. doi: $10.1002 / \mathrm{cncr} .31959$

146. Miyamoto DT, Zheng Y, Wittner BS, Lee RJ, Zhu H, Broderick $\mathrm{KT}$, et al. RNA-Seq of single prostate CTCs implicates noncanonical Wnt signaling in antiandrogen resistance. Science. (2015) 349:1351-6. doi: $10.1126 /$ science.aab0917 
147. Gao D, Vela I, Sboner A, Iaquinta PJ, Karthaus WR, Gopalan A, et al. Organoid cultures derived from patients with advanced prostate cancer. Cell. (2014) 159:176-87. doi: 10.1016/j.cell.2014.08.016

148. Borgmann H, Lallous N, Ozistanbullu D, Beraldi E, Paul N, Dalal K, et al. Moving towards precision urologic oncology: targeting enzalutamideresistant prostate cancer and mutated forms of the androgen receptor using the novel inhibitor darolutamide (ODM-201). Eur Urol. (2017) 16:e1290. doi: 10.1016/S1569-9056(17)30800-X

149. Au SH, Edd J, Stoddard AE, Wong KHK, Fachin F, Maheswaran S, et al. Microfluidic isolation of circulating tumor cell clusters by size and asymmetry. Sci Rep. (2017) 7:2433. doi: 10.1038/s41598-017-01150-3

150. Aceto N, Bardia A, Miyamoto DT, Donaldson MC, Wittner BS, Spencer JA, et al. Circulating tumor cell clusters are oligoclonal precursors of breast cancer metastasis. Cell. (2014) 158:1110-22. doi: 10.1016/j.cell.2014.07.013

151. Gkountela S, Castro-Giner F, Szczerba BM, Vetter M, Landin J, Scherrer R, et al. Circulating tumor cell clustering shapes DNA methylation to enable metastasis seeding. Cell. (2019) 176:98-112.e114. doi: 10.1016/j.cell.2018.11.046

152. Cellsearch ${ }^{\circledR}$ Circulating Tumor Cell Kit (Epithelial) Instructions for Use. South Raritan, NJ: Janssen Diagnostics, LLC.

153. Schott DS, Pizon M, Pachmann U, Pachmann K. Sensitive detection of PD-L1 expression on circulating epithelial tumor cells (CETCs) could be a potential biomarker to select patients for treatment with PD-1/PD-L1 inhibitors in early and metastatic solid tumors. Oncotarget. (2017) 8:7275572. doi: 10.18632 /oncotarget. 20346

154. Pachmann K, Schuster S. The value of monitoring the behavior of circulating tumor cells at the end of endocrine therapy in breast cancer patients. Cancers. 10:407. doi: 10.3390/cancers 10110407

155. Pizon M, Lux D, Pachmann U, Pachmann K, Schott D. Influence of endocrine therapy on the ratio of androgen receptor (AR) to estrogen receptor (ER) positive circulating epithelial tumor cells (CETCs) in breast cancer. J Transl Med. (2018) 16:356. doi: 10.1186/s12967-018-1724-Z

156. Su Z, Zhao J, Ke S, Zhang J, Liu X, Wang Y, et al. Clinical significance of circulating tumor cells via combined whole exome sequencing in early stage cancer screening: a case report. Exp Ther Med. (2018) 16:252733. doi: $10.3892 /$ etm. 2018.6507
157. Ge F, Zhang H, Wang DD, Li L, Lin PP. Enhanced detection and comprehensive in situ phenotypic characterization of circulating and disseminated heteroploid epithelial and glioma tumor cells. Oncotarget. (2015) 6:27049-64. doi: 10.18632/oncotarget. 4819

158. Golan T, Milella M, Ackerstein A, Berger R. The changing face of clinical trials in the personalized medicine and immuno-oncology era: report from the international congress on clinical trials in Oncology \& Hemato-Oncology (ICTO 2017). J Exp Clin Cancer Res. (2017) 36:192. doi: 10.1186/s13046-0170668-0

159. Parker CE, Borchers CH. The special issue: clinical proteomics for precision medicine. Proteomics Clin Appl. (2018) 12:1600144. doi: $10.1002 /$ prca.201600144

160. Steyer B, Cory E, Saha K. Developing precision medicine using scarless genome editing of human pluripotent stem cells. Drug Discov Today. (2018) 28:3-12. doi: 10.1016/j.ddtec.2018.02.001

161. Xi X, Li T, Huang Y, Sun J, Zhu Y, Yang Y, et al. RNA biomarkers: frontier of precision medicine for cancer. Non-Coding RNA. (2017) 3:9. doi: 10.3390/ncrna3010009

162. Mullard A. FDA approves landmark RNAi drug. Nat Rev Drug Discov. (2018) 17:613. doi: $10.1038 / \mathrm{nrd} .2018 .152$

163. Morrison C. Alnylam prepares to land first RNAi drug approval. Nat Rev Drug Discov. (2018) 17:156. doi: 10.1038/nrd. 2018.20

Conflict of Interest: The authors declare that the research was conducted in the absence of any commercial or financial relationships that could be construed as a potential conflict of interest.

Copyright (ㅇ) 2019 Malik, Srinivasan and Batra. This is an open-access article distributed under the terms of the Creative Commons Attribution License (CC BY). The use, distribution or reproduction in other forums is permitted, provided the original author(s) and the copyright owner(s) are credited and that the original publication in this journal is cited, in accordance with accepted academic practice. No use, distribution or reproduction is permitted which does not comply with these terms. 